\title{
LES ASPECTS AGRICOLES DU TTIP
}

Arthur Meert

\author{
CRISP | «Courrier hebdomadaire du CRISP »
}

2016/3 $n^{\circ} 2288$ | pages 5 à 50

ISSN 0008-9664

Article disponible en ligne à l'adresse :

https://www.cairn.info/revue-courrier-hebdomadaire-du-crisp-2016-3-page-5.htm

Distribution électronique Cairn.info pour CRISP.

(C) CRISP. Tous droits réservés pour tous pays.

La reproduction ou représentation de cet article, notamment par photocopie, n'est autorisée que dans les limites des conditions générales d'utilisation du site ou, le cas échéant, des conditions générales de la licence souscrite par votre établissement. Toute autre reproduction ou représentation, en tout ou partie, sous quelque forme et de quelque manière que ce soit, est interdite sauf accord préalable et écrit de l'éditeur, en dehors des cas prévus par la législation en vigueur en France. Il est précisé que son stockage dans une base de données est également interdit. 
Courrier hebdomadaire

$n^{\circ} 2288 \cdot 2016$

\section{Les aspects agricoles du TTIP}

Arthur Meert

(RISP 


\section{Courrier hebdomadaire}

Rédacteur en chef : Cédric Istasse

Assistante éditoriale : Fanny Giltaire

Le Courrier hebdomadaire est soutenu par l'Administration générale de l'Enseignement et de la Recherche scientifique de la Fédération Wallonie-Bruxelles. Il est également publié avec l'aide financière du Fonds de la recherche scientifique-FNRS.

Une version numérique du Courrier hebdomadaire est disponible en pay per view (au numéro) et en accès gratuit pour les abonnés sur le site portail de CAIRN (http://www.cairn.info).

Le numéro simple : 6,90 euros - le numéro double : 12,40 euros

Abonnement : 235,00 euros

Souscription, commandes et informations :

CRISP - Place Quetelet, 1A - 1210 Bruxelles

Tél : 32 (0)2 2110180 - Fax : 32 (0)2 2197934

http://www.crisp.be - info@crisp.be

IBAN BE51 310027157662 - Swift BBRUBEBB

Éditeur responsable : Jean Faniel - Place Quetelet, 1A - 1210 Bruxelles

Tous droits de traduction, d'adaptation ou de reproduction par tous procédés, y compris la photographie et le microfilm, réservés pour tous pays.

ISSN 00089664 


\section{TABLE DES MATIÈRES}

$\begin{array}{ll}\text { INTRODUCTION } & 5\end{array}$

1. LE CONTEXTE DE NÉGOCIATION DU TTIP $\quad 8$

1.1. D'une approche multilatérale à un projet bilatéral 8

1.2. Des modèles agricoles différents 9

1.3. Un projet en cours de négociation $\quad 12$

$\begin{array}{ll}\text { 1.4. Un projet controversé } & 13\end{array}$

2. LA LEVÉE DES BARRIÈRES TARIFAIRES 15

3. LA CONVERGENCE DES NORMES SANITAIRES ET PHYTOSANITAIRES ET LA LEVÉE DES OBSTACLES TECHNIQUES AU COMMERCE

3.1. Deux cas emblématiques

18

3.1.1. La convergence des normes sanitaires et phytosanitaires : le cas du bœuf aux hormones

La levée des obstacles techniques au commerce:

le cas des produits laitiers non pasteurisés $\quad 21$

3.2. Quelle convergence des normes sanitaires et phytosanitaires ? 22

3.3. L'établissement d'un système juridictionnel de protection des investissements 25

3.3.1. Le projet avorté d'ISDS 26

3.3.2. De l'ISDS au projet d'ICS 29

4. L'ÉTABLISSEMENT DE STANDARDS INTERNATIONAUX 31

CONCLUSION 34

\begin{tabular}{ll} 
ANNEXE & 37 \\
\hline
\end{tabular} 


\section{INTRODUCTION}

Le Partenariat transatlantique de commerce et d'investissement, mieux connu sous son nom anglais « Transatlantic Trade and Investment Partnership » (TTIP), est un projet d'accord de libre-échange entre l'Union européenne et les États-Unis d'Amérique ${ }^{1}$. Il a pour ambition de développer un marché commun en s'attaquant aux obstacles - tant tarifaires que non tarifaires - qui existent entre les deux partenaires, à travers trois objectifs principaux. Primo, il vise à diminuer les droits de douane et, de la sorte, à réduire les barrières tarifaires. Secundo, il cherche à parvenir à une plus grande cohérence des normes réglementaires des deux partenaires (voire à une harmonisation de celles-ci), afin d'optimiser les coûts administratifs liés à l'application de ces normes. Tertio, il insiste sur le développement d'une certaine coopération quant à la formulation de standards internationaux entre les partenaires, afin qu'ils puissent exploiter au maximum leur position de leaders économiques mondiaux ${ }^{2}$.

Le présent Courrier hebdomadaire étudie les implications que, s'il est adopté, le TTIP aurait pour un secteur spécifique : l'agriculture. En effet, cet aspect des négociations est de grande importance pour les deux partenaires. Du côté européen, la Politique agricole commune (PAC) est, depuis sa mise en application en 1962 (suite au Traité de Rome de 1957 instituant la Communauté économique européenne), un pilier de la politique européenne. Aux États-Unis, le marché agricole représente un pôle économique fondamental ${ }^{3}$. Bien que les échanges entre l'Union européenne et les États-Unis soient actuellement limités dans le secteur agricole - en comparaison, par exemple, avec la situation qui prévaut dans le secteur industriel ${ }^{4}-$, la mise en œuvre du TTIP pourrait avoir des répercussions importantes dans ce domaine. Par ailleurs, les aspects agricoles du TTIP - sanitaires et phytosanitaires, entre autres - sont souvent évoqués par les adversaires du projet de traité pour justifier leur position ; ainsi, les opposants européens dénoncent notamment le fait que la législation états-unienne accepte des pratiques telles que la culture ou l'élevage d'organismes génétiquement modifiés (OGM), le recours aux hormones dans la nourriture des bœufs ou le lavage des poulets au chlore. Sur la scène

1 Le TTIP ne doit être confondu ni avec le CETA (Comprehensive Trade and Economic Agreement), qui est un traité de libre-échange entre l'Union européenne et le Canada signé en septembre 2014 mais qui n'a pas encore été adopté, ni avec le TISA (Trade in Service Agreement), qui est un projet d'accord sur le commerce des services, négocié dans le cadre de l'OMC et qui inclut l'Union européenne, les États-Unis et 22 autres pays.

Commission européenne, Direction générale Commerce, «TTIP explained», 2014, http://trade.ec.europa.eu.

3 En 2013, seules la Chine et l'Inde comptaient un bénéfice net supérieur à celui des États-Unis dans le secteur agricole (respectivement 949 milliards de dollars pour la Chine et 336 milliards de dollars pour l'Inde, contre 226 milliards de dollars pour les États-Unis). Cf. Banque mondiale, «Agriculture, value added », s.d., http://data.worldbank.org.

4 En 2013, dans le secteur agricole, les importations dans l'Union européenne en provenance des ÉtatsUnis comptaient pour un total de 9,784 milliards d'euros, alors que les exportations de l'Union européenne vers les États-Unis pesaient 15,367 milliards d'euros. Par comparaison, dans le secteur industriel, la même année, les importations dans l'Union européenne en provenance des États-Unis s'élevaient à 185,499 milliards d'euros et les exportations de l'Union européenne vers les États-Unis à 272,389 milliards d'euros. Le commerce industriel était donc presque vingt fois plus développé que le commerce agricole. Cf. Commission européenne, Direction générale Commerce, «European Union, Trade in Goods with USA », 2014, http://trade.ec.europa.eu. 
belge francophone, le député européen Marc Tarabella (PS) a ravivé les questionnements liés à cette problématique en demandant, au début de l'année 2016, l'arrêt complet des négociations, au nom des dangers que celles-ci représentent à ses yeux pour l'agriculture européenne ${ }^{5}$.

Le TTIP étant encore en cours de négociation, il n'est pas possible à la société civile d'en connaitre le contenu. Outre, bien entendu, les négociateurs et leur entourage professionnel proche, seuls certains mandataires politiques (notamment, en Belgique, les parlementaires fédéraux et fédérés) ont accès à la version consolidée du texte, régulièrement mise à jour, mais ils sont tenus par des clauses strictes de confidentialité. Certes, la Commission européenne publie de nombreuses communications relatives au sujet. Mais il n'en reste pas moins que, à l'heure actuelle, le principal document officiel disponible est le mandat confié par les 28 États membres à la Commission européenne ; adopté le 14 juin 2013, il a été rendu public le 9 octobre $2014^{6}$.

Par ailleurs, diverses études sont disponibles. Relativement au domaine de l'agriculture, l'une des plus importantes d'entre elles est le rapport « Agriculture in the Transatlantic Trade and Investment Partnership: Tariffs, Tariff-Rate Quotas, and Non-Tariff Measures », publié en novembre 2015 par le Ministère de l'Agriculture des États-Unis (United States Department of Agriculture, USDA) ${ }^{7}$. Ce document envisage trois scénarios différents parmi les multiples cas de figure possibles : un premier dans lequel seules les barrières tarifaires seraient levées; un deuxième dans lequel, en outre, certaines barrières non tarifaires seraient harmonisées; et un troisième, similaire au deuxième, mais qui postule que les préférences des consommateurs iraient aux produits locaux, quel que soit le prix de ceux-ci. Sa conclusion est que, quel que soit le scénario qui prévaudra, les gains du TTIP seront bien plus avantageux pour le secteur agricole des États-Unis et pour l'économie états-unienne en général que pour le partenaire européen. Cette étude a été critiquée du côté européen, notamment par la commissaire au Commerce de la Commission Juncker, Cecilia Malmström, qui a souligné à plusieurs reprises qu'aucun des trois scénarios considérés ne correspondait à une situation susceptible d'être effective un jour ${ }^{8}$. Il n'empêche que, pour l'instant, il n'existe pas encore d'étude européenne similaire à celle de l'USDA ; un tel document est toutefois en cours de préparation ${ }^{9}$. Par ailleurs, en 2014 est parue une étude du Centre d'études prospectives et d'informations internationales (CEPII), commandée par la Commission de l'Agriculture et du Développement rural du Parlement européen ; intitulée « Risques et opportunités pour le secteur agroalimentaire européen liés à un possible accord commercial entre l'UE et les États-Unis », elle se concentre sur le secteur agroalimentaire ${ }^{10}$.

La Libre Belgique, 5 janvier 2016.

Ce document est reproduit en annexe de la présente publication.

J. BECKMAN et al., "Agriculture in the Transatlantic Trade and Investment Partnership: Tariffs, TariffRate Quotas, and Non-Tariff Measures », United States Department of Agriculture, Economic Research Service, novembre 2015, www.ers.usda.gov.

8 Cf. notamment ses déclarations devant les membres du Parlement wallon (Parlement wallon, Commission chargée des affaires européennes, Compte rendu intégral, CRIC 96, 29 janvier 2016, p. 17).

De même, le SPF Économie, PME, Classes moyennes et Énergie élabore actuellement une étude centrée sur la Belgique, notamment pour les aspects agricoles.

10 Centre d'études prospectives et d'informations internationales (CEPII), « Risques et opportunités pour le secteur agroalimentaire européen liés à un possible accord commercial entre l'UE et les États-Unis. Étude », Parlement européen, Direction générale des Politiques internes, 2014, www.europarl.europa.eu. 
Le premier chapitre de ce Courrier hebdomadaire situe le TTIP dans son contexte global. D'une part, il évoque la négociation des derniers cycles de l'Accord général sur les tarifs douaniers et le commerce (General Agreement on Tariffs and Trade, GATT) et l'activité de l'Organisation mondiale du commerce (OMC). D'autre part, il met en évidence les approches spécifiques qui caractérisent les politiques agricoles européenne et états-unienne selon trois critères: le degré de protection douanière, l'établissement des normes et la philosophie de culture agricole. Ce sont en effet les différences qui existent dans ces trois domaines qui expliquent les divergences d'appréciation quant aux implications potentielles du TTIP dans le secteur de l'agriculture.

Pour illustrer ce propos, les trois chapitres suivants analysent tour à tour les trois objectifs principaux identifiés dans le projet de TTIP, en montrant de quelle manière les différences évoquées ci-dessus peuvent affecter les deux partenaires. Le deuxième chapitre est consacré au thème de la levée des barrières tarifaires, et particulièrement des droits de douane. Le troisième chapitre analyse la problématique de la levée des barrières non tarifaires liées à la convergence des normes sanitaires et phytosanitaires, ainsi qu'aux obstacles techniques au commerce. Y est notamment envisagée la question du projet de système juridictionnel des investissements («investment court system», ICS) - qui remplace le projet avorté de mécanisme de règlement de différends entre investisseur et État («investor-to-state dispute settlement ", ISDS). Enfin, le quatrième chapitre aborde la question de l'établissement de standards internationaux à travers la question du droit de propriété intellectuelle, et particulièrement les indications géographiques.

La situation présentée est celle arrêtée à la mi-mars 2016. 


\section{LE CONTEXTE DE NÉGOCIATION DU TTIP}

La négociation du Partenariat transatlantique de commerce et d'investissement (Transatlantic Trade and Investment Partnership, TTIP) prend place dans un contexte où, bien que les deux partenaires adoptent des approches différentes en politique agricole, ils tentent de se positionner en leaders économiques mondiaux, en s'associant bilatéralement après l'échec de la négociation multilatérale qu'a constitué le cycle de Doha au début des années 2000. Replacer le TTIP dans ce contexte plus large permet de mieux en saisir les particularités.

\subsection{D'UNE APPROCHE MULTILATÉRALE À UN PROJET BILATÉRAL}

Les questions que soulève le projet de TTIP ne sont pas nouvelles dans les débats politiques internationaux relatifs à l'agriculture. Lorsque l'on examine le mandat de l'Accord général sur les tarifs douaniers et le commerce (General Agreement on Tariffs and Trade, GATT) lors de l'Uruguay Round (1986-1994), il apparaît que l'accès au marché, la réduction des barrières techniques aux échanges et les questions des normes sanitaires et phytosanitaires étaient alors déjà sur la table ${ }^{11}$. Par ailleurs, les accords de Marrakech qui ont conclu ce cycle de négociation ont débouché sur la création de l'Organisation mondiale du commerce (OMC), mais aussi sur la conclusion d'accords multilatéraux (qui ne concernent donc pas seulement l'Union européenne et les États-Unis) plus spécifiques sur l'agriculture : l'Accord sur l'agriculture de l'Uruguay Round (AAUR) et trois accords portant respectivement sur les mesures sanitaires et phytosanitaires (Sanitary and Phytosanitary, SPS), sur les obstacles techniques au commerce (Technical Barriers to Trade, TBT) et sur le droit de propriété intellectuelle lié au commerce (DPIC) ${ }^{12}$. De plus, l'agenda du cycle de Doha (2001-2006) - qui a échoué - comportait quelques sujets de l'actuel projet de TTIP, tels qu'un accès amélioré au marché, la question de la propriété intellectuelle (et particulièrement les indications géographiques, cf. infra) ou encore le règlement des différends.

Il est donc intéressant de constater que le projet de TTIP se place assez clairement dans le prolongement des négociations du GATT et de l'OMC. Cependant, à l'inverse des

11 A. BlogowsKI, V. BoRZeIX, «L'accord sur l'agriculture du cycle de l'Uruguay. Bilan et perspective pour l'Union européenne. Première partie », dans Ministère [français] de l'Agriculture et de la Pêche, Notes et études économiques, volume 13, Paris, 2001, p. 105-138.

Ibidem. 
accords du GATT et de l'OMC, le TTIP envisage une approche bilatérale. Cela reflète les problèmes qui, lors du cycle de Doha, ont été rencontrés en raison de l'adoption d'une approche multilatérale où un trop grand nombre d'acteurs aux intérêts trop divergents n'ont pu s'entendre ${ }^{13}$.

Une approche bilatérale doit permettre de négocier plus facilement, puisque le nombre d'acteurs, et donc d'exigences différentes, est réduit. Néanmoins - et c'est aussi un élément central pour bien comprendre les enjeux du TTIP -, les politiques agricoles de l'Union européenne et des États-Unis divergent à plusieurs égards. Ces différences apparaissent comme fondamentales pour expliquer les tensions qui entourent l'initiative, mais aussi, selon leurs promoteurs, les opportunités qui naîtraient de l'aboutissement de ces négociations.

\subsection{DES MODÈLES AGRICOLES DIFFÉRENTS}

Une première différence fondamentale entre les politiques agricoles menées de part et d'autre de l'Atlantique est liée au degré de protectionnisme que celles-ci admettent. En matière agricole, les États-Unis adoptent une approche du marché compétitive ${ }^{14}$. Même si certains secteurs, comme ceux du sucre ou du lait, sont protégés, la majorité des produits agricoles sont vendus selon les lois du marché. La politique agricole états-unienne est donc davantage offensive (visant à limiter toute barrière tarifaire pour permettre aux agriculteurs nationaux d'étendre leur marché au niveau international) que défensive (visant principalement à protéger ces agriculteurs par des barrières tarifaires). De son côté, depuis la création de la Politique agricole commune (PAC), l'Union européenne a insisté sur la "préférence communautaire ", qui consiste à favoriser les produits provenant des États membres au détriment des importations. C'est ce qui explique, à l'origine, la mise en place de tarifs douaniers élevés, ainsi que le développement de soutiens à la production pour les agriculteurs européens. Même si les dernières réformes tendent vers un degré de protectionnisme moins élevé qu'auparavant et que les soutiens directs à la production ont été réduits voire supprimés, les taxes à l'importation restent en moyenne deux fois plus élevées dans l'Union européenne qu'aux États-Unis.

Les deux politiques agricoles peuvent également être distinguées par leur approche dans l'établissement des normes sanitaires et phytosanitaires. Alors que l'Union européenne s'appuie sur le principe de précaution et met en ouvre un contrôle « de la ferme à la table ", les États-Unis ont une approche «basée sur la science ».

Le principe de précaution est cité pour la première fois dans la législation européenne à l'occasion du Traité de Maastricht de 1992. À l'origine, ce principe n'était d'application que dans le domaine de la protection de l'environnement (article 130R). En 2000, la Commission l'a élargi à la santé humaine, animale et végétale. Ce principe est d'application lorsqu' « une évaluation scientifique objective et préliminaire indique qu'il est raisonnable

13 A. PONTVIANNE, «L'agriculture à l'OMC : libéralisation, développement et souveraineté », in L'agriculture, nouveaux défis, Paris, Institut national de la statistique et des études économiques, 2007, p. 123-142.

E. Trachtenberg, «A Transatlantic Partnership: Agricultural Issues. Different Visions, a Common Destiny », Economic Policy Paper Series, German Marshall Fund of the United States, octobre 2012, p. 7. 
de craindre les effets potentiellement dangereux pour l'environnement ou la santé humaine, animale ou végétale ${ }^{15}$ - de tels effets potentiels étant estimés " incompatibles avec le niveau élevé de protection choisi par la Commission ${ }^{16}$. Pour que le principe de précaution soit d'application, il faut donc que soient identifiés des effets potentiellement négatifs. Plus précisément, il n'est pas nécessaire que soient produites des preuves scientifiques absolues de dangerosité, mais seulement que soit mise en évidence une « incertitude scientifique » relative à l'absence de dangerosité. C'est sur la base de ce principe que, par exemple, la culture ou l'élevage d'organismes génétiquement modifiés (OGM) ou l'utilisation d'hormones dans l'engraissage du bétail ont été interdites dans l'Union européenne ${ }^{17}$.

Quant à lui, le contrôle « de la ferme à la table » garantit que « chaque maillon de la chaîne alimentaire, de la production primaire jusqu'à la vente au détail, [soit] couvert par la législation de l'UE en matière de sécurité alimentaire " ${ }^{18}$. Exprimée dans le «Livre blanc sur la sécurité alimentaire » du 12 janvier $2000^{19}$, cette volonté européenne de traçabilité des produits agricoles a été concrétisée par les législations européennes de 2002 (législation " de la ferme à la table ») ${ }^{20}$ et de 2004 ( « paquet hygiène ») ${ }^{21}$. Ses origines remontent à la fin des années 1980, époque marquée à la fois par une volonté d'ouverture des frontières intérieures de l'Europe et par la crise de la vache folle. Afin d'éviter tout risque de propagation de maladies animales, des systèmes de traçabilité et de contrôle sanitaire ont alors été mis en place au niveau européen pour le transport des animaux et pour l'alimentation animale ${ }^{22}$.

De leur côté, les États-Unis privilégient une approche «basée sur la science », c'est-à-dire selon laquelle des normes doivent être posées, non par précaution, mais uniquement suite à la démonstration scientifique de l'existence d'un risque avéré, objectivé, observé ${ }^{23}$. Il est à noter que, sur cette question, les accords de l'OMC suivent l'approche étatsunienne. En effet, ils prévoient qu'« une mesure sanitaire ou phytosanitaire ne soit

15 Commission européenne, "Communication de la Commission sur le recours au principe de précaution », 2 février 2000, p. 2, http://europa.eu.

16 Ibidem.

17 E. FABRY, G. GARBASSO, «La réalité de la précaution : analyse comparée des réglementations du risque aux États-Unis et en Europe (éd. J. Wiener, M. Rogers, J. Hammitt et P. Sand) », Notre Europe, Institut Jaques Delors, 18 juillet 2014, www.notre-europe.eu.

18 Commission européenne, Direction générale Santé et Protection des consommateurs, 50 ans de sécurité alimentaire dans l'Union européenne, 1957-2007, Luxembourg, Office des publications officielles des Communautés européennes, 2007, p. 16, http://ec.europa.eu.

19 Commission européenne, «Livre blanc sur la sécurité alimentaire », 12 janvier 2000, http://ec.europa.eu.

Règlement (CE) no 178/2002 du Parlement européen et du Conseil du 27 janvier 2002 établissant les principes généraux et les prescriptions générales de la législation alimentaire, instituant l'Autorité européenne de sécurité des aliments et fixant les procédures relatives à la sécurité des denrées alimentaires, Journal officiel des Communauté européennes, L 31, $1^{\text {er }}$ février 2002.

21 Ensemble de plusieurs règlements du Parlement et du Conseil du 29 avril 2004, dont le règlement (CE) $\mathrm{n}^{\circ} 852 / 2004$ relatif à l'hygiène des denrées alimentaires, le règlement (CE) n $\mathrm{n}^{\circ}$ 853/2004 fixant des règles spécifiques d'hygiène applicables aux denrées alimentaires d'origine animale, le règlement $(\mathrm{CE})$ $n^{\circ} 854 / 2004$ fixant les règles spécifiques d'organisation des contrôles officiels concernant les produits d'origine animale destinés à la consommation humaine et le règlement $(\mathrm{CE}) \mathrm{n}^{\circ}$ 882/2004 relatif aux contrôles officiels effectués pour s'assurer de la conformité avec la législation sur les aliments pour animaux et les denrées alimentaires et avec les dispositions relatives à la santé animale et au bien-être des animaux (Journal officiel de l'Union européenne, L 139, 30 avril 2004 et L 191, 28 mai 2004).

22 Commission européenne, Direction générale Santé et Protection des consommateurs, 50 ans de sécurité alimentaire dans l'Union européenne, 1957-2007, op. cit.

Ibidem, p. 1. 
appliquée que dans la mesure nécessaire pour protéger la santé et la vie des personnes et des animaux ou pour préserver les végétaux, qu'elle soit fondée sur des principes scientifiques et qu'elle ne soit pas maintenue sans preuves scientifiques suffisantes ${ }^{24}$. Dans le domaine agricole ${ }^{25}$, l'Union européenne est donc systématiquement plus stricte que les États-Unis en termes de mesures de précaution ${ }^{26}$.

Une troisième et dernière différence fondamentale entre les politiques agricoles des deux partenaires réside dans la philosophie de production. Au cours des vingt dernières années, l'Union européenne a adopté une approche multifonctionnelle de l'agriculture. Cette approche prend en compte l'impact que l'agriculture peut avoir au niveau tant environnemental que social ${ }^{27}$. Insufflée par la réforme McSharry opérée en $1992^{28}$, elle a progressivement amené à mettre en avant divers aspects non économiques, comme le développement rural ou la protection des zones agricoles défavorisées. Comparativement à celle adoptée aux États-Unis, cette approche européenne s'attache davantage à maintenir une agriculture multifonctionnelle, produisant à un niveau élevé de qualité, et familiale, parfois même dans le respect de certaines traditions ${ }^{29}$. À l'inverse, la philosophie étatsunienne pourrait plutôt être qualifiée d'industrielle. On compte aux États-Unis 2,2 millions de fermes, contre 7,9 millions dans l'Union européenne. En moyenne, une ferme étatsunienne couvre 169 hectares, soit sept fois plus qu'une ferme européenne. Enfin, on dénombre environ 3 millions d'habitants des États-Unis employés dans le secteur, alors que plus de 16 millions de citoyens européens travaillent dans l'agriculture. Les fermes étatsuniennes sont généralement plus rentables parce qu'elles ont recours à des technologies nouvelles optimisant la production, qu'elles sont plus grandes et qu'elles emploient proportionnellement moins de main-d'œuvre.

Tableau 1. Caractéristiques comparées des agricultures européenne et états-unienne

\begin{tabular}{|l|l|l|}
\cline { 2 - 3 } \multicolumn{1}{c|}{} & \multicolumn{1}{c|}{ Union européenne } & \multicolumn{1}{c|}{ États-Unis } \\
\hline Degré de protectionnisme & $\begin{array}{l}\text { Tendanciellement en diminution } \\
\text { mais «préférence communautaire » }\end{array}$ & $\begin{array}{l}\text { Approche offensive, peu de taxes } \\
\text { de douane }\end{array}$ \\
\hline Établissement des normes & $\begin{array}{l}\text { Principe de précaution et contrôle } \\
\text { «de la ferme à la table » }\end{array}$ & Approche «basée sur la science » \\
\hline Philosophie de production & $\begin{array}{l}\text { Multifonctionnelle, parfois familiale } \\
\text { et traditionnelle }\end{array}$ & Industrielle \\
\hline
\end{tabular}

24 Organisation mondiale du commerce (OMC), "Accord sur l'application des mesures sanitaires et phytosanitaires », article 2.2, s.d., www.wto.org.

25 Tel n'est pas le cas dans tous les secteurs, comme par exemple celui des médicaments (cf. J. B. WIENER et al. (dir.), The Reality of Precaution: Comparing Risk Regulation in the United States and Europe, Washington/Londres, RFF Press, 2011).

26 Une exception notable est toutefois à signaler : lors de la crise la vache folle, les États-Unis ont réagi rapidement, d'une part, en interdisant les importations de viande bovine et, d'autre part, en refusant tout don de sang émanant d'une personne ayant vécu minimum trois mois au Royaume-Uni, mesure maintenue jusqu'en 1996 (cf. E. FABRY, G. GARBASSO, «La réalité de la précaution », op. cit., p. 4-5).

27 G. C. ALONS, «The TAFTA | TTIP and Agriculture: Making or Breaking the Tackling of Global Food and Environmental Challenges? ", in D. CARDOSO et al. (dir.), The Transatlantic Colossus. Global Contributions to Broaden the Debate on the EU-US Free Trade Agreement, Nimègue, Radboud University, Institute for Management Research, 2014, p. 63-67, https://futurechallenges.org.

28 Sur cette réforme, cf. T. GARCIA AZCARATE, «La politique agricole commune : situation actuelle et développements futurs prévisibles », 1996, http://tomasgarciaazcarate.com.

29 E. Trachtenberg, «A Transatlantic Partnership », op. cit., p. 8. 


\subsection{UN PROJET EN COURS DE NÉGOCIATION}

En novembre 2011, lors du sommet entre l'Union européenne et les États-Unis, il est décidé de mettre en place un groupe d'experts (High Level Working Group on Jobs and Growth, HLWG), chargé d'identifier « des mesures pour augmenter le commerce et les investissements entre l'Union européenne et les États-Unis afin de mutuellement soutenir la création d'emplois, la croissance économique et la compétitivité internationale ${ }^{30}$. En février 2013, ce groupe remet un rapport qui constitue les bases du projet de TTIP ${ }^{31}$. Le document pointe trois éléments principaux, qui seraient selon ses rédacteurs les fondements d'un marché transatlantique global.

Le premier est l'accès au marché pour les investisseurs et les entreprises. Il est suggéré que différents droits de douane soient supprimés et que le secteur des services soit libéralisé, au même titre que celui des investissements.

Le deuxième point concerne la réduction des barrières non tarifaires, et particulièrement la question des normes et des régulations. Le rapport du HLWG souligne l'importance de mettre en place un système commun qui permettrait de maintenir un niveau de régulation élevé, tout en évitant que les complexités administratives liées à l'application de ces normes sur les deux continents n'entravent le développement des entreprises. Les normes européennes et états-uniennes sont considérées comme très proches sur le fond mais très différentes sur la forme, situation qui complique le commerce entre les deux rives de l'Atlantique. Cette observation conduit le HLWG à préconiser la reconnaissance mutuelle et l'harmonisation des régulations. Selon le rapport, l'établissement de normes communes élargirait le marché des entreprises européennes et états-uniennes et optimiserait les coûts administratifs liés à l'application de ces normes.

Le troisième point dépasse les seules relations bilatérales entre l'Union européenne et les États-Unis. Le rapport met en avant l'importance commerciale que pourrait représenter un marché transatlantique dans son ensemble : jouissant d'un statut de leaders mondiaux, les deux partenaires devraient travailler à établir des règles et standards applicables internationalement. Il est ainsi suggéré de se concentrer sur certains domaines comme la propriété intellectuelle, la protection des travailleurs ou l'environnement.

En février 2013, le président de la Commission européenne, José Manuel Barroso, le président du Conseil européen, Herman Van Rompuy, et le président des ÉtatsUnis, Barack Obama, annoncent conjointement que, sur la base du rapport du HLWG, les négociations relatives au TTIP sont lancées ${ }^{32}$.

Actuellement, ces tractations sont toujours en cours. La Commission européenne négocie au nom des 28 États membres de l'Union européenne ${ }^{33}$, qui l'ont mandatée pour remplir

30 High Level Working Group on Jobs and Growth (HLWG), «Final Report », 11 février 2013, http://trade.ec.europa.eu.

31 Ibidem.

32 Commission européenne, «Statement from United States President Barack Obama, European Council President Herman Van Rompuy and European Commission President José Manuel Barroso », 13 février 2013, http://europa.eu.

33 Les négociations relatives à la politique commerciale de l'Union européenne relèvent de la compétence exclusive de la Commission européenne ; celle-ci ne peut cependant négocier des accords de politique commerciale que sur la base de mandats qui lui sont confiés à l'unanimité par les États membres. 
cette tâche, et avec l'aval du Parlement européen ${ }^{34}$. Le mandat de négociation relatif au TTIP a été délivré à la Commission européenne par le Conseil des affaires étrangères (Questions commerciales) le 14 juin 2013. Selon la procédure habituelle dans un tel cadre, la Commission européenne rend régulièrement compte de l'état d'avancement des négociations aux États membres et consulte les parties concernées ("stakeholders»). Du côté états-unien, c'est le Bureau du représentant au Commerce des États-Unis (United States Trade Representative, USTR) qui est chargé de mener les négociations ${ }^{35}$.

Douze "cycles de négociations", c'est-à-dire douze périodes de rencontres et de négociations entre les représentants de la Commission et ceux de l'USTR, se sont déjà tenus, alternativement en Europe (à savoir à Bruxelles) et aux États-Unis (le plus souvent à Washington, mais également à New York et à Miami). Le négociateur en chef du TTIP pour l'Union européenne est Ignacio Garcia Bercero.

Lorsqu'un accord final aura été obtenu entre les deux partenaires, il devra être avalisé, du côté européen, par le Conseil des ministres (c'est-à-dire par les gouvernements respectifs de chaque État membre de l'Union européenne) et par le Parlement européen ${ }^{36}$ et, du côté états-unien, par le Congress (Senate et House of Representatives) ${ }^{37}$. En outre, si l'accord est considéré comme mixte - c'est-à-dire comme touchant à des compétences qui ne relèvent pas exclusivement du ressort de l'Union européenne (à savoir, dans le cas présent, le commerce international) mais également des États membres -, il devra être ratifié en Europe par les différents parlements nationaux (ainsi que, dans le cas des États fédéraux, par les parlements des entités fédérées). À ce stade, il est probable mais pas assuré que le TTIP se verra reconnaître le statut de traité mixte.

\subsection{UN PROJET CONTROVERSÉ}

Rapidement, l'opacité des négociations est vivement critiquée de l'extérieur. Une mobilisation importante de la société civile européenne (et, dans une moindre mesure, de la société civile états-unienne $)^{38}$ se développe en opposition au projet de TTIP. Elle

34 Résolution du Parlement européen du 23 mai 2013 sur les négociations en vue d'un accord en matière de commerce et d'investissement entre l'Union européenne et les États-Unis, www.europarl.europa.eu.

35 Membre de la branche exécutive du gouvernement fédéral des États-Unis, le représentant au Commerce est en charge de la politique commerciale internationale du pays. Ce poste est occupé depuis 2013 par Michael Froman.

36 Commission européenne, Direction générale Commerce, "Faire du TTIP une réalité », s.d., http://ec.europa.eu.

7 I. FERGUSON, «Trade Promotion Authority (TPA) and the Role of Congress in Trade Policy », Congress Research Service, 15 juin 2015, p. 9, https://fas.org.

38 Selon le journaliste Jean-François Gérard, la société civile états-unienne se mobilise généralement moins pour critiquer des traités de libre-échange comme le TTIP ; de plus, aux États-Unis, le débat est plus porté sur l'Accord de partenariat transpacifique (Trans-Pacific Partnership Agreement, traité multilatéral de libre-échange signé le 4 février 2016 et visant à intégrer les économies des régions Asie-Pacifique et Amérique) que sur le TTIP (cf. J.-F. GÉRARD, «Traité de libre-échange : qu'en pensent les États-Unis ? », Arte, 2014). Il existe néanmoins des organisations de la société civile états-unienne qui se sont mobilisées (cf., par exemple, Public Citizen, "The Trans-Atlantic "Free Trade" Agreement (TAFTA)», s.d., www.citizen.org/tafta). 
prend notamment la forme d'une initiative citoyenne européenne ${ }^{39}$ baptisée «Stop TTIP ». Entre octobre 2014 et octobre 2015, celle-ci recueille plus de 3 millions de signatures de citoyens européens émanant des 28 pays de l'Union. Toutefois, dès septembre 2014, la Commission européenne rejette cette initiative. Pour justifier cette position, elle indique n'être pas compétente dans le domaine contesté, mais être simplement mandatée par les États membres pour les représenter (cf. supra) ${ }^{40}$.

La pression ainsi mise sur les institutions européennes conduit néanmoins les trois principales d'entre elles à réagir (Conseil de l'Union européenne, Commission européenne et Parlement européen). En octobre 2014, le Conseil de l'Union européenne rend public le contenu du mandat de négociation confié à la Commission. Bien que plus détaillé que les suggestions du HLWG, ce document semble suivre fidèlement celles-ci.

En mai 2015, la commissaire européenne en charge du Commerce, Cecilia Malmström, plaide pour une adaptation du mécanisme projeté de règlement de différends entre investisseur et État ( «investor-to-state dispute settlement », ISDS) ${ }^{41}$, afin de revoir l'équilibre des rapports dans un sens plus favorable aux États et moins avantageux pour les investisseurs ; elle avance l'idée de recourir plutôt à une "international investment court » (cf. infra). Cette proposition vise clairement à répondre aux critiques soulevées au sein de la société civile et du Parlement européen vis-à-vis de l'ISDS. Le mois suivant, le Parlement européen, où de nombreuses voix critiquent à la fois le fond (le recours à l'ISDS et un risque de nivellement vers le bas) et la forme (l'opacité des négociations) du projet de TTIP, approuve une recommandation quant aux garanties que cette assemblée désire recevoir pour avaliser le projet. Ce texte, qui n'a aucune valeur contraignante, plaide notamment pour un maintien des normes sanitaires et sociales, ainsi que pour une protection des services publics en général par rapport à des fournisseurs privés qui pourraient les concurrencer ${ }^{42}$.

On le voit, le projet de TTIP est très controversé. L'analyse des impacts potentiels d'un tel traité sur le secteur agricole, à laquelle se livre la suite de la présente étude, constitue également l'occasion de mettre en évidence quelques-uns des arguments des opposants et des défenseurs du projet. En l'occurrence, trois aspects principaux du projet de TTIP en cours de négociation sont susceptibles d'avoir des répercussions dans le domaine agricole: la levée des barrières tarifaires, la convergence des normes sanitaires et phytosanitaires et la levée des obstacles techniques au commerce, et l'établissement de standards internationaux.

39 L'initiative citoyenne européenne est un instrument introduit par le Traité de Lisbonne, mis à la disposition des citoyens européens depuis 2012. Une demande rassemblant au moins un million de signatures dans minimum sept pays de l'Union européenne permet de mettre une problématique à l'agenda de la Commission européenne.

40 Commission européenne, Secrétariat général, «Your request for registration of a proposed citizens' initiative entitled "STOP TTIP" ", 10 septembre 2014, http://ec.europa.eu.

C. MALMSTRÖM, "Investments in TTIP and Beyond. Towards an International Investment Court», 5 mai 2015, http://ec.europa.eu.

42 Résolution du Parlement européen du 8 juillet 2015 contenant les recommandations du Parlement européen à la Commission européenne concernant les négociations du partenariat transatlantique de commerce et d'investissement (PTCI), 2015, www.europarl.europa.eu. 


\section{LA LEVÉE DES BARRIÈRES TARIFAIRES}

Le mandat publié par le Conseil de l'Union européenne et sur la base duquel la Commission mène les négociations avec les États-Unis prévoit :

«L'accord aura pour but de supprimer l'ensemble des droits sur les échanges bilatéraux. L'objectif commun sera la suppression d'un grand nombre de droits de douane au moment de l'entrée en vigueur de l'accord, suivie de la suppression progressive de tous les droits de douane, à l'exception des plus sensibles, à brève échéance. Lors des négociations, les deux parties examineront des options pour le traitement des produits les plus sensibles, y compris les contingents tarifaires. Tous les droits de douane, taxes, prélèvements ou redevances à l'exportation ainsi que les restrictions quantitatives ou les exigences en matière d'autorisation concernant les exportations vers l'autre partie qui ne sont pas justifiés par des exceptions dans le cadre de l'accord seront supprimés au moment de l'entrée en vigueur de l'accord. Les négociations s'attacheront à répondre aux préoccupations concernant les obstacles restants au commerce des biens à double usage qui portent atteinte à l'intégrité du marché unique. »

Concrètement, selon les hypothèses du Centre for Economic Policy Research (CEPR) - dont une étude, commandée par la Commission européenne, constitue le document scientifique de référence de celle-ci -, la réduction des droits de douane serait de l'ordre de $98 \%{ }^{43}$. S'il est adopté, le projet de traité entend donc supprimer quasi totalement les barrières tarifaires. Selon la Commission européenne, ce volet du TTIP ne posera pas de problèmes, eu égard au fait que le niveau des tarifs douaniers entre les deux partenaires est déjà très bas ${ }^{44}$.

Cependant, plusieurs auteurs remettent cette affirmation en question ${ }^{45}$. Selon eux, les politiques agricoles respectives des deux partenaires font que l'Union européenne serait la grande perdante d'une levée (quasi) complète des droits de douane sur les produits agricoles. En effet, ces droits sont plus élevés dans l'Union européenne qu'aux États-Unis, même s'ils ont certes connu une réduction importante lors des négociations du GATT, depuis le Dillon Round de 1960 jusqu'à l'Uruguay Round (1986-1994) ${ }^{46}$, puis dans le cadre des réformes de la PAC menées au cours des décennies 1990 et 2000. Il est tout

Centre for Economic Policy Research (CEPR), « Reducing Transatlantic Barriers to Trade and Investment: An Economic Assessment. Final Project Report », mars 2013, p. 2, http://trade.ec.europa.eu.

${ }_{44}$ Commission européenne, "Questions fréquentes », s.d., http://ec.europa.eu.

45 Cf. notamment T. Josling, S. Tangermann, "Agriculture, Food and the TTIP: Possibilities and Pitfalls ", Center for European Policy Studies/Center fort Transatlantic Relations, décembre 2014, https://papers.ssrn.com; J. BERTHELOT, «Les aspects tarifaires agricoles du TAFTA et de l'APE Afrique de l'Ouest », Les Possibles, $n^{\circ}$ 4, 15 septembre 2014, https://france.attac.org.

46 A. BlogowsKi, V. BORZEIX, «L'accord sur l'agriculture du cycle de l'Uruguay », op. cit. 
aussi vrai que les tarifs douaniers entre l'Union européenne et les États-Unis sont actuellement assez bas : les produits agricoles importés sont taxés, en moyenne, à hauteur de $11 \%$ dans l'Union européenne et de 4,5\% aux États-Unis ${ }^{47}$. Certains secteurs jouissent d'un statut spécifique qui leur permet de maintenir un niveau de protection élevé afin de maintenir, en Europe, la fameuse préférence communautaire. À l'heure actuelle, les secteurs les plus protégés par la PAC sont, entre autres, ceux des produits laitiers (avec une taxe de $45 \%$ en moyenne, pouvant s'élever à $134 \%$ dans certains cas spécifiques), des fruits et légumes (en moyenne $10 \%$, pouvant aller jusqu'à $182 \%$ ) et du sucre (en moyenne $26 \%$, pouvant aller jusqu'à $94 \%$ ). Du côté des États-Unis, bien que le marché soit presque totalement libéralisé dans ce pays, deux secteurs importants restent protégés : ceux des produits laitiers (taxés en moyenne à $17 \%$, parfois jusqu'à $188 \%$ ) et du sucre (en moyenne $12 \%$, pouvant aller jusqu'à $55 \%)^{48}$. Pour ces secteurs, la levée des droits de douane pourrait s'avérer particulièrement problématique ${ }^{49}$, puisqu'ils sont les plus protégés avant la mise en œuvre d'une réduction telle que celle prévue par le projet de TTIP. L'Union européenne protégeant en moyenne davantage ses productions agricoles que les États-Unis, ce serait donc ceux-ci qui profiteraient d'une plus grande augmentation de leurs exportations.

L'étude publiée en novembre 2015 par le Ministère de l'Agriculture des États-Unis (United States Department of Agriculture, USDA) semble confirmer cette hypothèse. En effet, dans le premier scénario qu'elle envisage, où seules les barrières tarifaires seraient levées, les gains pour les États-Unis en termes d'exportations de produits agricoles seraient presque sept fois plus importants que ceux de l'Union européenne (respectivement 5,5 milliards de dollars contre à peine 800 millions de dollars $)^{50}$. Au niveau mondial, une telle libéralisation représenterait même une perte de 0,25\% des exportations de l'Union européenne, mais une hausse de $2 \%$ de celles des États-Unis ${ }^{51}$. Cependant, comme l'a indiqué C. Malmström (notamment devant le Parlement wallon), un tel scénario semble inenvisageable ${ }^{52}$. De plus, la Commission européenne a précisé ceci en 2015 : «Là où la suppression immédiate des droits de douane européens pourrait poser problème, nous voulons convenir d'une suppression progressive qui laisserait aux entreprises de l'UE le temps de s'adapter. Et si, malgré cette suppression progressive, les entreprises de l'UE risquaient malgré tout de connaître des difficultés, nous n'ouvririons que partiellement notre marché $"{ }^{53}$.

Au-delà de cette question, il est nécessaire de considérer à la fois les aspects tarifaires et les aspects non tarifaires du projet de TTIP pour saisir le plus complètement possible l'impact potentiel de celui-ci. Cela est d'autant plus vrai que, sans la levée des obstacles non tarifaires (cf. infra), la réduction des droits de douane n'aurait qu'une incidence limitée. L'étude commandée par la Commission européenne au CEPR estime que la seule

\footnotetext{
OMC, « United States and European Union Tariff Profile », 2014, http://stat.wto.org. Ibidem.

T. Josling, S. TANGermanN, « Agriculture, Food and the TTIP », op. cit., p. 4-5.

J. BECKMAN et al., « Agriculture in the Transatlantic Trade and Investment Partnership », op. cit., p. 12. Ibidem, p. 16.

Parlement wallon, Commission chargée des affaires européennes, Compte rendu intégral, CRIC 96, 29 janvier 2016.

53 Commission européenne, Direction générale Commerce, Au cœur du TTIP. Le partenariat transatlantique de commerce et d'investissement (TTIP). Vers un accord commercial Union européenne - États-Unis. Un tour d'horizon et un guide chapitre par chapitre, dans un langage clair, Luxembourg, Office des publications de l'Union européenne, 2015, p. 12, http://trade.ec.europa.eu.
} 
réduction des tarifs douaniers (sans prendre en compte la levée des barrières non tarifaires, donc) réduirait l'impact potentiel du TTIP de quelque $60 \%{ }^{54}$. Pour sa part, le Centre d'études prospectives et d'informations internationales (CEPII) confirme cette supposition ; il estime même que « la libéralisation des droits de douane n'expliquerait qu'un quart des incidences totales " ${ }^{55}$. Même si ses résultats doivent être interprétés prudemment, l'étude de l'USDA va dans le même sens : elle considère que, dans le scénario où certaines barrières non tarifaires seraient également levées ou harmonisées, l'Union européenne et les États-Unis pourraient chacun plus ou moins doubler leurs bénéfices (ils réaliseraient des gains supplémentaires de, respectivement, 1,2 milliard de dollars et 4,1 milliards de dollars $)^{56}$. De plus, comme le rappellent différentes études liées à l'impact de la levée d'une taxe à l'importation ${ }^{57}$, une telle opération n'a guère d'intérêt si, malgré tout, des barrières non tarifaires continuent à compliquer voire à empêcher l'entrée d'un produit sur un marché. La Commission européenne insiste elle aussi sur la nécessité de réduire les barrières non tarifaires afin de réduire les coûts du commerce entre l'Union européenne et les États-Unis.

54 CEPR, «Reducing Transatlantic Barriers to Trade and Investment», op. cit., p. 95. Cette étude évalue les gains qui seraient réalisés par l'Union européenne à un montant situé entre 68 et 119 milliards d'euros, selon les scénarios envisagés (avec une moyenne de 93 milliards d'euros), et ceux des États-Unis à entre 50 et 95 milliards d'euros (avec une moyenne de 72 milliards d'euros). Les gains résultant seulement d'une réduction des tarifs douaniers seraient ramenés à, respectivement, 24 et 9 milliards d'euros. Le fait que l'étude du CEPR prévoie des gains supérieurs pour l'Union européenne que pour les États-Unis, alors que celle de l'USDA arrive à la conclusion inverse, s'explique par le fait que la première étude envisage tous les secteurs du TTIP alors que la seconde se concentre sur les seuls aspects agricoles.

55 CEPII, «Risques et opportunités pour le secteur agroalimentaire européen liés à un possible accord commercial entre l'UE et les États-Unis », op. cit., p. 41.

56 J. BECKMAN et al., "Agriculture in the Transatlantic Trade and Investment Partnership », op. cit., p. 28.

57 CEPII, « Risques et opportunités pour le secteur agroalimentaire européen liés à un possible accord commercial entre l'UE et les États-Unis », op. cit. ; E. TRACHTENBERG, «A Transatlantic Partnership », op. cit. 


\section{LA CONVERGENCE DES NORMES SANITAIRES ET PHYTOSANITAIRES ET LA LEVÉE DES OBSTACLES TECHNIQUES AU COMMERCE}

L'OMC définit comme "barrière non tarifaire » tout obstacle, autre qu'une taxe à proprement parler, de type administratif, technique ou juridique, et dont l'application ralentit ou diminue les possibilités de commerce entre deux partenaires économiques ${ }^{58}$. Dans le cas du projet de TTIP, la justification de la levée de telles barrières est simple : les systèmes normatifs des deux partenaires sont présentés comme similaires sur le fond - ils assurent des niveaux équivalents de protection des consommateurs et de l'environnement - mais très différents sur la forme - ils obligent les entreprises exportatrices à se conformer à des critères différents via des procédures administratives différentes (cf. supra). L'objectif recherché par la réduction des barrières non tarifaires est de réduire les coûts administratifs résultant de l'emploi de deux systèmes de régulation différents en harmonisant ces derniers (convergence des normes), tout en maintenant un niveau de protection de la santé et de l'environnement suffisant pour tous ${ }^{59}$.

Ainsi, le mandat confié à la Commission européenne par le Conseil de l'Union européenne dispose :

« L'accord visera à supprimer, par des mécanismes efficaces, les obstacles superflus au commerce et aux investissements, y compris les obstacles non tarifaires en vigueur, en parvenant à un niveau ambitieux de compatibilité réglementaire pour les biens et les services, y compris par la reconnaissance mutuelle, l'harmonisation et le renforcement de la coopération entre régulateurs. La compatibilité réglementaire ne fera pas obstacle au droit de réglementer en fonction du niveau de protection de la santé, de la sécurité, des consommateurs, des travailleurs, de l'environnement et de la diversité culturelle que chaque partie juge approprié, ou de manière à atteindre des objectifs réglementaires légitimes (...). À cet effet, l'accord comprendra des dispositions portant [notamment] sur [les] mesures sanitaires et phytosanitaires (mesures SPS), [les] réglementations techniques, normes et procédures d'évaluation de la conformité, [et la] cohérence réglementaire.»

Ce mandat de négociation insiste donc, entre autres, sur deux points : d'une part, la nécessité de faire converger les normes sanitaires et phytosanitaires (qui concernent la préservation de l'environnement, ainsi que le bien-être et le respect humain, animal

58 OMC, « Obstacles non tarifaires : lourdeurs administratives, etc. », s.d., www.wto.org.

9 HLWG, «Final Report », op. cit., p. 3. 
et végétal, cf. supra) ${ }^{60}$ et, d'autre part, l'importance de conclure un accord sur les obstacles techniques au commerce (c'est-à-dire sur les mesures qui, indépendamment des normes sanitaires et phytosanitaires, comprennent tout règlement technique ou administratif auquel il convient de se conformer) ${ }^{61}$.

Ces deux points sont particulièrement importants pour comprendre le projet de TTIP - dans le domaine agricole comme dans de nombreux autres. Tandis que l'importance du protectionnisme permet de saisir les enjeux liés à la levée des barrières tarifaires (cf. supra), c'est la manière d'établir les normes qui est ici au centre de la problématique. Au vu des nombreux articles publiés sur la question ${ }^{62}$ et de la mobilisation de la société civile européenne à ce sujet ${ }^{63}$, on peut estimer que cette question est un des nœuds du débat sur ce projet de traité transatlantique.

On a vu plus haut qu'il existe une différence entre l'Union européenne et les États-Unis quant à la manière d'établir des normes : la première applique le principe de précaution (qui permet d'établir des normes sur la base de l'absence de preuve de non-dangerosité) et un contrôle " de la ferme à la table » (qui garantit un respect de ces normes à toutes les étapes de production), tandis que les seconds adoptent une approche «basée sur la science» (des normes ne sont imposées que si est apportée la preuve scientifique d'un danger pour le consommateur ou l'environnement). Une telle différence de situation s'explique par la relative confiance des citoyens vis-à-vis de leurs organes de contrôle sanitaire respectifs. En effet, selon Eric Trachtenberg, expert états-unien spécialisé dans les questions agricoles et d'économie internationale, et qui a notamment travaillé à l'USDA, les producteurs et consommateurs états-uniens sont traditionnellement assez confiants envers les administrations sanitaires de leur pays, qu'ils estiment compétentes et transparentes. À l'inverse, selon lui, l'influence de crises alimentaires comme celle de la vache folle ou de la dioxine a rendu les Européens plus méfiants par rapport à leur système de régulation de santé publique et aux nouveautés introduites en la matière, ce qui explique une approche plus prudente ${ }^{64}$.

\subsection{DeUX CAS EMblématiQueS}

Le cas du bœuf aux hormones et celui des produits laitiers non pasteurisés permettent d'illustrer l'impact que pourrait avoir, respectivement, une convergence des normes sanitaires et phytosanitaires et une levée des obstacles techniques au commerce.

60 L. D. TAmini, R. Dupuis, M. Doyon, «Commerce international des produits alimentaires. Capacité d'application de la réciprocité des normes (innocuité, environnement et social) et leviers à la disposition des gouvernements. Rapport de projet », Centre interuniversitaire de recherche en analyse des organisations, octobre 2014, p. 30, www.cirano.qc.ca.

$61 \quad$ Ibidem, p. 32.

62 Cf., par exemple, M. vom ENDT, «Is TAFTA | TTIP a Race to the Bottom in Regulatory Standards? The Case of Hormone-Treated Beef ", in D. CARDOso et al. (dir.), The Transatlantic Colossus, op. cit., p. 99-103 ; E. TraChTENBERG, «A Transatlantic Partnership », op. cit.

63 Cf., par exemple, Corporate Europe Observatory (CEO), «An Open Door for GMOs? Take Action on the EU-US Free Trade Agreement », 22 mai 2013, http://corporateeurope.org.

64 E. TrachtenberG, «A Transatlantic Partnership », op. cit., p. 8-9. 


\subsubsection{La convergence des normes sanitaires et phytosanitaires : le cas du bouf aux hormones}

Suite à différents scandales liés aux hormones en Europe, le Conseil des ministres européen de l'Agriculture a décidé, en 1988, de bannir six hormones du marché européen au nom du principe de précaution. Cette décision a reçu le soutien du Parlement européen. Les États-Unis, ainsi que d'autres pays producteurs de bœufs aux hormones, qui se sentent défavorisés injustement par cette mesure qu'ils estiment protectionniste, ont attaqué l'Union européenne devant l'OMC. En 1998, celle-ci a donné raison aux États-Unis, au motif que l'interdiction de ces produits n'était pas basée sur des faits scientifiques et qu'il existait un large consensus quant à l'absence de dangers que représente l'utilisation de ce genre d'hormones dans la nourriture des bœufs. L'Union européenne a toutefois préféré payer aux États-Unis la somme de 125 millions de dollars par an plutôt que d'accepter la production et l'importation de viande à base d'hormones ${ }^{65}$.

Ce cas met deux constats en lumière. D'une part, il montre bien la différence d'approche de l'Union européenne et des États-Unis quant aux normes sanitaires et phytosanitaires dans le domaine agricole : les seconds sont très favorables à l'utilisation de nouveaux produits pour augmenter le rendement, tandis que la première, craignant un danger potentiel des hormones, se refuse complètement à les accepter, malgré une pénalité financière significative ${ }^{66}$. D'autre part, la décision de l'OMC souligne que l'approche "basée sur la science " prévaut lors de conflits économiques de ce type.

À l'instar de celui des poulets lavés au chlore ou de celui de la culture ou de l'élevage d'OGM, le cas du bœuf aux hormones est devenu le cheval de bataille des plus fervents opposants au projet de TTIP. Selon eux, ces différents cas illustrent la philosophie générale qu'ils perçoivent dans le projet de TTIP : servir l'intérêt des entreprises états-uniennes dans l'ouverture des marchés, au détriment des consommateurs et des agriculteurs européens.

Cependant, la Commission européenne met en avant le fait que ces pratiques agricoles controversées et les productions qui en sont issues ne risquent en aucun cas d'être imposées aux États membres de l'Union européenne sans leur accord. En effet, ces cas polémiques sont exclus par le mandat de négociation, qui indique :

« L'accord devrait reconnaître que le développement durable est l'un des objectifs principaux des parties et que ces dernières s'efforceront de garantir et de faciliter le respect des normes et accords internationaux en matière d'environnement et de travail, tout en favorisant de hauts niveaux de protection de l'environnement, des travailleurs et des consommateurs, conformément à l'acquis de l'UE et à la législation des États membres. L'accord devrait établir que les parties ne favoriseront pas les échanges ou les investissements directs étrangers en réduisant la portée de la législation et des normes internes en matière d'environnement, d'emploi ou de santé et sécurité au travail, ou en assouplissant les normes fondamentales du travail ou les politiques et la législation visant à protéger et à promouvoir la diversité culturelle »;

65 W. A. KerR, J. E. HobBS, « The North American-European Union Dispute over Beef Produced Using Growth Hormones: A Major Test for the New International Trade Regime », World Economy, n 25, 2002, p. 283-296.

66 E. TrachtenberG, «A Transatlantic Partnership », op. cit. 
«Les dispositions du chapitre consacré aux [mesures sanitaires et phytosanitaires (SPS)], développeront les principes essentiels de l'accord de l'OMC sur les mesures SPS, y compris l'exigence en vertu de laquelle les mesures SPS de chaque partie doivent reposer sur une base scientifique et sur des normes internationales ou sur des évaluations des risques de nature scientifique, tout en reconnaissant le droit des parties à apprécier et gérer les risques conformément au niveau de protection que chaque partie juge approprié, en particulier lorsque les preuves scientifiques pertinentes sont insuffisantes, mais doivent n'être appliquées que dans la mesure nécessaire pour protéger la santé et la vie des personnes et des animaux ou préserver les végétaux, et doivent être élaborées de manière transparente, sans retard indu. »

\subsubsection{La levée des obstacles techniques au commerce : le cas des produits laitiers non pasteurisés}

De plus, à l'inverse de ce que suggèrent certains opposants, la réduction des barrières non tarifaires pourrait aussi profiter aux Européens. Ainsi, l'étude réalisée par le CEPII met en avant le secteur du lait européen, pour lequel seraient bénéfiques une réforme en termes d'harmonisation des normes sanitaires et phytosanitaires et une réduction des obstacles techniques au commerce ${ }^{67}$. En effet, l'importation de ces produits aux ÉtatsUnis est soumise à des règles strictes d'origine et de composition, ainsi qu'à des règles administratives qui rendent les importations européennes quasiment impossibles. De ce fait, une réduction ou une révision de ces obstacles techniques au commerce serait potentiellement bénéfique pour le secteur européen des produits laitiers, en particulier celui des fromages. Cet avantage qualitatif permettrait de développer largement les exportations aux États-Unis ${ }^{68}$.

Au niveau des réglementations en matière sanitaire et phytosanitaire, l'analyse du CEPII est néanmoins plus nuancée quant à l'apport positif que le TTIP pourrait avoir pour le secteur européen. En effet, la vente de produits à base de lait cru (non pasteurisé) n'est guère autorisée aux États-Unis, ce qui limite extrêmement les exportations vers ce pays de fromages européens produits à base de lait cru tels le brie, le camembert ou le roquefort. Or, le CEPII estime qu'il n'est « guère probable que les États-Unis facilitent les importations [de produits à base de lait cru], eu égard aux craintes des associations [états-uniennes] de consommateurs, qui ont rassemblé des éléments scientifiques solides en faveur de la pasteurisation obligatoire ${ }^{69}$.

Le cas des produits laitiers non pasteurisés met donc, lui aussi, en avant plusieurs éléments importants. Il montre que, potentiellement, la levée des obstacles techniques au commerce sur les produits laitiers pourrait être bénéfique pour les agriculteurs, en particulier européens puisque c'est pour eux que les règles s'assoupliraient. Cela pourrait donc

67 CEPII, « Risques et opportunités pour le secteur agroalimentaire européen liés à un possible accord commercial entre l'UE et les États-Unis ", op. cit., p. 57.

68 J. GRUEFF, «Achieving a Successful Outcome for Agriculture in the EU-US Transatlantic Trade and Investment Partnership Agreement », International Food \& Agricultural Trade Policy Council, février 2013, p. 17, www.agritrade.org.

CEPII, «Risques et opportunités pour le secteur agroalimentaire européen liés à un possible accord commercial entre l'UE et les États-Unis », op. cit., p. 57. 
être un exemple concret d'apport positif du TTIP pour l'Union européenne. Cependant, les normes sanitaires et phytosanitaires états-uniennes relatives au lait, jugées trop sévères par les Européens, ne seront probablement pas assouplies, vu la base scientifique sur laquelle elles reposent. Par conséquent, l'effet potentiellement positif du premier aspect pourrait être annihilé par le second.

\subsection{QUELLE CONVERGENCE DES NORMES SANITAIRES ET PHYTOSANITAIRES?}

Les deux cas que nous venons de développer semblent indiquer qu'une levée des obstacles techniques au commerce pourrait être mutuellement bénéfique (particulièrement, pour l'Union européenne, dans le secteur laitier). Néanmoins, la question de la convergence des normes sanitaires et phytosanitaires semble, quant à elle, être plus problématique pour le partenaire européen. En effet, dans les deux cas développés ci-dessus, les normes états-uniennes semblent prévaloir - celles-ci suivant les principes énoncés par l'OMC (cf. supra) -, tandis que l'application du principe de précaution auquel recourt l'Union européenne pourrait être remise en question. Aussi, la convergence des normes sanitaires et phytosanitaires apparait-elle comme potentiellement très problématique pour le marché européen, à la fois pour des raisons économiques et pour des motifs sanitaires.

En ce qui concerne la convergence de ces normes, deux scénarios semblent envisageables : soit une reconnaissance mutuelle des normes - situation dans laquelle les producteurs européens et états-uniens resteraient soumis à leurs propres règles, tout en partageant un marché commun -, soit une véritable harmonisation des normes - dans ce cas, les producteurs des deux côtés de l'Atlantique seraient soumis aux mêmes règles ${ }^{70}$.

Ici également, l'exemple du bœuf aux hormones est intéressant à évoquer pour faire comprendre la problématique. En effet, il est régulièrement utilisé par les opposants pour illustrer leurs craintes. Et ce, bien que la Commission européenne a insisté à plusieurs reprises sur le fait, d'une part, que ce cas de figure est exclu des négociations (cf. supra) et, d'autre part, que le but du TTIP sera d'harmoniser les normes à niveau égal voire supérieur (et que, dès lors, un cas comme celui du bœuf aux hormones ne sera donc simplement pas envisageable) ${ }^{71}$.

La reconnaissance mutuelle des normes sanitaires et phytosanitaires impliquerait que l'importation de bœuf aux hormones états-unien serait autorisée en Europe alors que les producteurs européens devraient toujours se soumettre au principe de précaution et ne pourraient donc élever, pour leur part, que des bœufs sans hormones. Économiquement, cela engendrerait une situation de concurrence déloyale, puisque la production de viande bovine avec hormones permet un rendement supérieur de $40 \%$ à une production sans

\footnotetext{
T. Josling, S. TANGermann, «Agriculture, Food and the TTIP », op. cit., p. 14.

Cf., par exemple, Commission européenne, Direction générale Commerce, 10 idées reçues sur le TTIP. Le partenariat transatlantique de commerce et d'investissement. Démêler le vrai du faux, Luxembourg, Office des publications de l'Union européenne, 2015, http://trade.ec.europa.eu; Parlement wallon, Commission chargée des affaires européennes, Compte rendu intégral, CRIC 96, 29 janvier 2016, p. 20.
} 
hormones ${ }^{72}$. Les producteurs européens ne seraient plus du tout concurrentiels face à leurs homologues états-uniens. Par ailleurs, les consommateurs européens seraient touchés, d'un point de vue sanitaire, par l'entrée en Europe de produits ne respectant pas les normes européennes. De la même manière, mais dans le sens inverse, les produits laitiers européens pourraient affecter le marché états-unien.

Les implications d'une véritable harmonisation des normes seraient différentes. Pour les entreprises concernées, cela représenterait des millions d'euros ou de dollars d'économies ${ }^{73}$. En outre, une telle situation éviterait une situation de concurrence déloyale en tant que telle, tous les producteurs étant alors soumis à des règles communes de part et d'autre de l'Atlantique. Mais cela impliquerait de décider quelles normes appliquer, en particulier en matière sanitaire. Les plus farouches opposants à l'harmonisation des mesures sanitaires redoutent à cet égard un «nivellement par le bas " ${ }^{74}$, qui consisterait à aligner les normes européennes sur les règles états-uniennes. Selon eux, le but ultime du projet de TTIP, et notamment de son volet agricole, est de servir les intérêts de l'agrobusiness en abaissant les standards en vigueur dans l'Union européenne. L'observatoire des lobbies en Europe, le Corporate Europe Observatory (CEO), appuie une telle affirmation sur l'existence de courriers électroniques par lesquels la Direction générale du Commerce de la Commission européenne a sollicité Monsanto, multinationale de l'agro-industrie, pour des conseils quant à l'harmonisation des mesures en matière agricole $^{75}$. Un autre argument est avancé pour expliquer les raisons des velléités supposées de nivellement par le bas : en cas d'harmonisation des normes, soit les États-Unis devront augmenter la sévérité de leurs règles, soit l'Union européenne devra abaisser la sévérité des siennes. Or, certains experts estiment probable que ce sera la deuxième solution qui s'imposera, eu égard au fait que les normes européennes, généralement jugées trop draconiennes par les États-Unis, constituent autant d'obstacles au commerce (tel est, par exemple, le cas de l'interdiction du bœuf aux hormones).

Si l'on se réfere aux normes états-uniennes, il semble cependant inexact de parler de nivellement systématique vers le bas. En effet, bien que les normes en matière d'hormones soient plus strictes dans l'Union européenne qu'elles ne le sont aux États-Unis, tel n'est pas le cas pour tous les produits, comme l'illustre le cas des produits à base de lait non pasteurisé. Il est dès lors plus adéquat de parler d'une tendance à l'alignement sur les normes états-uniennes, comme on a pu l'observer dans les deux cas étudiés. En effet, même si les normes états-uniennes sont parfois plus strictes que les normes européennes, elles suivent la philosophie de l'OMC (cf. supra). Pareille approche est d'ailleurs également soutenue par la Chambre de commerce des États-Unis (United States Chamber of Commerce), mais aussi par l'organisation patronale BusinessEurope ${ }^{76}$. Un tel alignement donnerait à court terme un avantage économique aux entreprises états-uniennes, déjà

72 J. BERTHELOT, «Les aspects tarifaires agricoles du TAFTA et de l'APE Afrique de l'Ouest », op. cit.; CEPII, « Risques et opportunités pour le secteur agroalimentaire européen liés à un possible accord commercial entre l'UE et les États-Unis ", op. cit., p. 67. Précisons cependant que cette réduction des coûts de production n'est pas seulement due à l'utilisation d'hormones, mais aussi aux différences entre les fermes européennes et états-uniennes envisagées supra.

M. VOM ENDT, "Is TAFTA | TTIP a Race to the Bottom in Regulatory Standards? », op. cit., p. 100.

Ibidem ; CEO, « TTIP: A Lose-Lose Deal for Food and Farming », 8 juin 2014, http://corporateeurope.org.

CEO, «Busting the Myths of Transparency around the EU-US Trade Deal», 25 septembre 2014, http://corporateeurope.org.

76 M. vOM ENDT, «Is TAFTA | TTIP a Race to the Bottom in Regulatory Standards? », op. cit. 
adaptées à ces régulations ${ }^{77}$. Cette dernière considération semble d'autant plus envisageable que la Commission européenne elle-même explique que, à court terme, certains secteurs européens de la viande pourraient être touchés négativement par la mise en place du $\operatorname{TTIP}^{78}$.

Tableau 2. Impact potentiel des deux scénarios possibles de convergence des normes sanitaires et phytosanitaires

\begin{tabular}{|l|l|l|}
\cline { 2 - 3 } \multicolumn{1}{c|}{} & \multicolumn{1}{c|}{ Aspects économiques } & \multicolumn{1}{c|}{ Aspects sanitaires } \\
\hline Reconnaissance mutuelle & Concurrence déloyale & $\begin{array}{l}\text { Absence de contrôle sanitaire selon } \\
\text { des critères locaux sur les produits } \\
\text { importés }\end{array}$ \\
\hline $\begin{array}{l}\text { Harmonisation des normes } \\
\text { (vers les normes états-uniennes) }\end{array}$ & $\begin{array}{l}\text { Coûts d'adaptation aux normes } \\
\text { pour les entreprises européennes }\end{array}$ & $\begin{array}{l}\text { Alignement sur les normes états- } \\
\text { uniennes }\end{array}$ \\
\hline
\end{tabular}

Le cas hypothétique du bœuf aux hormones illustre bien les craintes que certaines personnes éprouvent quant aux dangers potentiels qu'une convergence des normes pourrait représenter pour les consommateurs européens et pour l'agriculture européenne. Pour sa part, la Commission européenne le récuse, en expliquant : « Nous maintiendrons nos niveaux élevés de protection. Dans un certain nombre de domaines, les réglementations européennes et américaines assurent une protection tout aussi rigoureuse et pourraient être compatibles. Si ce n'est pas le cas, nous garderons nos niveaux de protection respectifs ${ }^{79}$. Il n'empêche que cet exemple (comme celui des produits laitiers non pasteurisés) semble montrer que ce sont surtout les normes sanitaires et phytosanitaires (et pas les obstacles techniques au commerce) qui pourraient poser problème pour les Européens, en cas de convergence réglementaire (puisque, dans les deux cas, l'alignement se ferait sur l'approche de l'OMC).

Plus largement, le cas du bœuf aux hormones est emblématique des difficultés rencontrées dans le débat au TTIP. Ainsi, même si la Commission européenne se montre désireuse de donner une image de clarté et de transparence (autant que faire se peut dans le cadre de la négociation d'un tel traité international), force est de reconnaître que le mandat qui lui a été confié par le Conseil de l'Union européenne est ambivalent. Ainsi, comme déjà mentionné, ce mandat reconnaît « le droit des parties à apprécier et gérer les risques conformément au niveau de protection que chaque partie juge approprié, en particulier lorsque les preuves scientifiques pertinentes sont insuffisantes", précise que "la compatibilité réglementaire ne fera pas obstacle au droit de réglementer en fonction du niveau de protection de la santé, de la sécurité, des consommateurs, des travailleurs, de l'environnement (...) que chaque partie juge approprié » et indique «que les parties ne favoriseront pas les échanges ou les investissements directs étrangers en réduisant la portée de la législation et des normes internes en matière d'environnement (...) ou de santé ». À la lecture de ces extraits, il apparaît que des cas comme celui du bœuf aux hormones, qui concernent des produits qui ne sont pas soumis à des réglementations

77 J. BERTHELOT, « Les aspects tarifaires agricoles du TAFTA et de l'APE Afrique de l'Ouest », op. cit.

Commission européenne, Direction générale Commerce, «Transatlantic Trade and Investment Partnership.

The Economic Analysis Explained ", septembre 2013, http://trade.ec.europa.eu.

Commission européenne, Direction générale Commerce, Au cœur du TTIP, op. cit., p. 19. 
équivalentes sur le fond de part et d'autre de l'Atlantique, ne seront jamais concernés par le TTIP.

Néanmoins, il est intéressant de noter que, avant de reconnaître « le droit des parties à apprécier et gérer les risques conformément au niveau de protection que chaque partie juge approprié », le mandat précise que « les dispositions du chapitre consacré aux mesures SPS développeront les principes essentiels de l'accord OMC sur les mesures SPS, y compris l'exigence en vertu de laquelle les mesures SPS de chaque partie doivent reposer sur une base scientifique et sur des normes internationales ou des évaluations de risque de nature scientifique » (cf. supra). La position de la Commission européenne semble donc balancer entre une volonté de laisser une certaine liberté aux partenaires quant à leurs manières d'établir des normes et une volonté d'imposer à ceux-ci que les normes reposent sur des bases scientifiques, similairement à l'approche de l'OMC. Or, l'affaire qui a opposé l'Union européenne face aux États-Unis sur le cas du bœuf aux hormones illustre l'incompatibilité de ces deux positions (cf. supra).

\subsection{L'ÉTABLISSEMENT D'UN SYSTĖME JURIDICTIONNEL DE PROTECTION DES INVESTISSEMENTS}

Cette crainte d'incompatibilité se manifeste aussi dans un des aspects les plus polémiques du TTIP : celui de l'établissement d'un tribunal d'arbitrage visant à régler les différends entre investisseur et État.

Dans son volet relatif à l'«accès au marché », le mandat confié à la Commission européenne par le Conseil de l'Union européenne fait référence à un mécanisme de règlement des différends entre investisseur et État (appelé, en anglais, système de "investorto-state dispute settlement », ISDS). Ce mécanisme vise à assurer aux investisseurs étrangers un traitement standardisé, assuré par un tribunal privé et basé sur plusieurs critères leur assurant une protection accrue de leurs investissements. Parmi ces critères, figure l'assurance de recevoir un traitement juste et équitable ; le cas échéant, l'investisseur a le droit de réclamer des compensations ${ }^{80}$. En résumé, ce mécanisme permet aux entreprises de s'opposer à des régulations établies dans des pays où elles ont investi, sur la base du fait que ces régulations nuiraient au développement de leurs affaires. Concrètement, si un investisseur dont le siège se situe dans un État membre de l'Union européenne ou aux États-Unis se sent discriminé par une règle établie par un autre État concerné par le TTIP et dans lequel il a investi, il peut invoquer l'ISDS. Trois avocats - nommés l'un par l'investisseur, l'autre par l'État attaqué et le dernier d'un commun accord des deux parties (ou à défaut, par le Centre international pour le règlement des différends relatifs aux investissements, CIRDI, qui est un organe de la Banque mondiale) sont chargés de déterminer si l'État incriminé a ou non enfreint une disposition du TTIP, de telle sorte que l'investisseur concerné a été discriminé.

$80 \quad$ Ibidem, p. 9. 


\subsubsection{Le projet avorté d'ISDS}

Ainsi, le mandat prévoit l'établissement d'un "règlement des différends entre les investisseurs et l'État (RDIE) ». Il indique que le TTIP devra "garantir le niveau de protection et de sécurité juridique le plus élevé possible pour les investisseurs européens aux États-Unis" et "instaurer des conditions de concurrence équitables pour les investisseurs aux États-Unis et dans l'UE », en incluant notamment "un traitement juste et équitable, comportant l'interdiction de mesures déraisonnables, arbitraires ou discriminatoires». Il précise notamment que l'accord devra être "sans préjudice du droit de l'UE et des États membres d'adopter et d'appliquer, conformément à leurs compétences respectives, les mesures nécessaires pour poursuivre de manière non discriminatoire des objectifs légitimes de politique publique, par exemple en ce qui concerne le domaine social, l'environnement, la sécurité, la stabilité du système financier, la santé et la sécurité publiques ». Ces derniers mots traduisent une certaine ambiguïté de l'ISDS, de même qu'une certaine imprécision quant à la faculté qu'un tel système laisserait aux États membres de maintenir un niveau de régulation élevé.

À ce propos, les cas de Phillip Morris contre l'Uruguay et de Lone Pine contre le Canada illustrent comment un mécanisme similaire à l'ISDS peut être activé.

En 2006, Philip Morris, multinationale spécialisée dans la production et la commercialisation du tabac et dont le siège est établi à Lausanne, a attaqué l'Uruguay dans le cadre d'un accord de libre-échange existant entre ce pays et la Suisse. L'affaire a été portée devant un tribunal de règlement des différends similaire à celui proposé dans le cadre du projet de TTIP $^{81}$. La multinationale critiquait les mesures prises par l'État uruguayen afin d'imposer des emballages neutres pour les paquets de cigarettes. Bien que de telles mesures se soient avérées efficaces pour réduire la consommation de tabac et, donc, pour améliorer la santé publique, Philip Morris jugeait ces mesures discriminatoires et en opposition avec l'idée d'un traitement juste et équitable. La firme a dès lors réclamé 25 millions de dollars à l'Uruguay pour compenser les pertes que ces mesures lui auraient engendrées ${ }^{82}$.

Dans le cadre de l'accord de libre-échange entre les États-Unis, le Canada et le Mexique (ALENA), Lone Pine, une entreprise états-unienne opérant dans le secteur de l'extraction de gaz et de pétrole, a réclamé 250 millions de dollars de compensation au Canada. Pour attaquer le Canada, l'entreprise a eu recours à un tribunal de règlement des différends pour investisseurs; elle s'est appuyée sur la clause liée à la protection des investisseurs pour critiquer la mise en place par le gouvernement de la Province du Québec d'un moratoire relatif aux dangers environnementaux liés aux nouvelles techniques d'extraction de ces deux matières premières ${ }^{83}$. Selon Lone Pine, une telle décision représenterait

Pour plus de détails, cf. les articles 1117 et 1126 du Traité ALENA.

M. C. Porterfield, C. R. Byrnes, «Philip Morris v. Uruguay: Will Investor-State Arbitration Send Restrictions on Tobacco Marketing Up in Smoke? », International Institute for Sustainable Development, 12 juillet 2011, www.iisd.org.

83 CEO, «The Right to Say No: EU-Canada Trade Agreement Threatens Fracking Bans », 6 mai 2013, http://corporateeurope.org. 
une « révocation arbitraire (...) et illégale de [son] droit fondamental de prospecter du gaz et du pétrole ${ }^{84}$.

Quel que soit l'aboutissement de ces deux litiges, du fait qu'ils font référence à un système assez proche de l'ISDS prévu par le projet de TTIP, ils illustrent bien les implications que peut avoir ce type de mécanisme de règlement des différends entre investisseur et État. Tout d'abord, les deux cas se placent dans le contexte d'un accord de libre-échange. Ensuite, les termes précis utilisés par les firmes lorsqu'elles ont porté plainte sont des expressions qui se retrouvent explicitement dans le mandat publié à l'initiative $d u$ Conseil de l'Union européenne : "mesures irraisonnables » et atteinte au droit à « un traitement juste et équitable » dans le cas de Philip Morris ${ }^{85}$, traitement «arbitraire » dans celui de Lone Pine. Enfin, les deux procédures concernent des politiques dont l'objectif est, a priori, de servir l'intérêt commun à travers la protection de la santé publique ou de l'environnement.

Sur la base de cette comparaison, il semble imaginable que, avec un mécanisme tel que l'ISDS projeté, une entreprise comme Monsanto, par exemple, qui vend des OGM et défend la thèse de l'innocuité de tels produits, puisse attaquer les mesures protectrices liées à ce type de culture prises par l'Union européenne ou par l'un de ses États membres, au motif qu'elles soient discriminatoires et qu'elles nuisent à ses profits.

Différents acteurs individuels ou collectifs, comme le CEO, se sont mobilisés pour dénoncer les conséquences négatives que pourrait avoir un tel mécanisme de règlement des différends ${ }^{86}$. Tout d'abord, il ne pourrait être invoqué qu'unilatéralement (puisque les États n'auraient pas la possibilité de porter plainte contre les investisseurs), ce qui favoriserait les investisseurs au détriment des États ${ }^{87}$. Ensuite, l'ISDS limiterait l'action des gouvernements et parlements dans les États concernés par le TTIP. Enfin, ce genre d'actions en justice étant intéressantes financièrement, non seulement pour des plaignants qui obtiendraient gain de cause mais aussi pour les bureaux d'avocats qui les représenteraient, on peut s'attendre à une élévation du nombre de plaintes dans de nombreux domaines. D'une part, cela pourrait avoir un coût financier important pour les États qui auraient à se défendre. D'autre part, et par conséquent, on pourrait s'attendre à ce que ces États se montrent plus prudents au moment de légiférer dans le sens d'une protection de l'environnement et des consommateurs, par crainte d'être ensuite attaqués et de perdre in fine du temps et de l'argent. Ces craintes sont d'ailleurs confirmées par le rapport du CEPII, qui relève que, comme « certains règlements environnementaux (et éventuellement en matière de sécurité alimentaire également) pourraient être contestés, les secteurs agricole et alimentaire sont (...) concernés ${ }^{88}$. Greenpeace ajoute que le fait que de nombreux avocats soient, parallèlement à leur fonction au barreau, impliqués dans

\footnotetext{
«Arbitrary (...) and illegal revocation of [its] valuable right to mine for oil and gas» (ibidem).

M. C. Porterfield, C. R. BYrNes, « Philip Morris v. Uruguay », op. cit.

CEO, "The Right to Say No ", op. cit.

J.-C. GAROT, «Ầ qui profite le crime? » [interview de Thierry Bodson, secrétaire général de l'interrégionale wallonne de la FGTB], Pour écrire la liberté, 25 mai 2015, www.pour.press.

88 CEPII, «Risques et opportunités pour le secteur agroalimentaire européen liés à un possible accord commercial entre l'UE et les États-Unis », op. cit., p. 75.
} 
le monde des affaires provoquera sûrement des conflits d'intérêt, des avocats étant amenés à être à la fois juges et parties ${ }^{89}$.

Sur la base de ces différentes observations, les opposants européens au projet de TTIP font valoir que, dans le secteur agro-alimentaire, la mise en place de l'ISDS aurait pour tendance de faire prévaloir des normes sanitaires et phytosanitaires se rapprochant de celles prévues par l'OMC - celles des États-Unis, donc - et empêcherait l'Union européenne de continuer à appliquer le principe de précaution.

Il est important de noter que l'ISDS n'est pas propre au projet de TTIP. Il s'agit là d'un mécanisme qui a été créé en 1959, dans le cadre d'un traité bilatéral entre l'Allemagne et le Pakistan. Depuis lors, il a été inclus dans de nombreux autres accords commerciaux (dont 86 concernent la Belgique), sans que - a fait valoir la commissaire européenne C. Malmström devant le Parlement fédéral belge - cela ait posé de problème jusqu'à présent ${ }^{90}$. S'appuyant sur un rapport de la Conférence des Nations unies sur le commerce et le développement (CNUCED), le Centre national de coopération au développement (CNCD-11.11.11, ONG belge) indique cependant qu'un nombre grandissant de plaintes est enregistré : 568 plaintes déposées entre 2000 et 2013, contre 50 entre 1965 et $2000^{91}$.

Quoi qu'il en soit, l'opposition grandissante de la société civile pousse la Commission européenne à organiser une consultation publique à ce sujet entre mars et juillet 2014. Cette consultation confirme les craintes émises par la société civile et les ONG. La Commission note cependant qu'elle a pu établir que $97 \%$ des réponses recueillies consistaient en réalité en de simples « copier-coller » de réponses types proposées par différentes $\mathrm{ONG}^{92}$. Similairement, elle constate que $97 \%$ des réponses émanent de sept pays (à savoir, par ordre décroissant de répondants, le Royaume-Uni, l'Autriche, l'Allemagne, la France, la Belgique, les Pays-Bas et l'Espagne), ce qui permet de situer plus précisément l'opposition à l'ISDS - et, par extension, au TTIP - sur un plan géographique.

À l'occasion de cette consultation populaire, plusieurs critiques ont été mises en avant ${ }^{93}$. Beaucoup de répondants perçoivent l'ISDS comme constituant un danger pour les services publics, qui seront mis en concurrence avec des fournisseurs privés. Ils voient aussi dans ce tribunal un potentiel «épouvantail » pour la démocratie, les États pouvant devenir plus timorés à l'idée de légiférer. Par ailleurs, ils s’interrogent sur la nécessité d'un tel mécanisme dans une situation où tous les partenaires autour de la table ont des systèmes juridiques forts et indépendants. Plus encore, de nombreuses critiques pointent le potentiel contournement des juridictions nationales qu'un tel mécanisme permettrait. Mettant

89 M. HOLBACH, P. SCHOCKWEILER, «L'accord de libre échange : “TTIP, une menace qui nous concerne tous !" ", avril 2014, Greenpeace Luxembourg, p. 9, www.greenpeace.org. Cf. aussi A. ZACHARIE, N. VAN NufFEL, M. CERMAK, "Traité transatlantique (TTIP) : cartographie d'un partenariat controversé », Point Sud, les études du CNCD-11-11-11, juin 2015, p. 20, www.cncd.be.

90 Maison du peuple d'Europe, «Audition de Madame Cecilia Malmström, commissaire européenne au Commerce auprès de la commission des Relations extérieures du Parlement fédéral de Belgique, le 12 mai 2015 », 22 mai 2015 http://mpe-hve.be.

91 Conférence des Nations unies sur le commerce et le développement (CNUCED), « Trade and Development Report 2014. Global Governance and Policy Space for Development », 2014, p. 139 (cité par A. ZACHARIE, «Quand les intérêts des firmes priment sur les choix démocratiques des États », 3 juillet 2015, www.cncd.be).

92 Commission européenne, «Report. Online public consultation on investment protection and investorto-state dispute settlement (ISDS) in the Transatlantic Trade and Investment Partnership Agrement (TTIP) », 13 janvier 2015, p. 3, http://trade.ec.europa.eu.

93 Ibidem, p. 14-17. 
en cause la transparence du processus et l'indépendance des juges, ils estiment qu'un tel tribunal représenterait un mécanisme favorisant les investisseurs aux dépens des États.

Quoique minoritaires, quelques éléments en faveur de l'ISDS ont également été récoltés ${ }^{94}$ à commencer par l'impact positif qu'un tel mécanisme pourrait avoir sur les investissements étrangers et donc sur la croissance et l'emploi. Par ailleurs, certains répondants ont mis en avant les craintes qu'ils nourrissent à l'égard de la juridiction états-unienne publique. Enfin, un dernier argument évoqué pour s'opposer à toute abolition de l'ISDS tel qu'il est proposé est qu'il n'existe aucune preuve de l'impact négatif d'un tel mécanisme.

Même si l'on ne peut évidemment pas se fier aux chiffres de cette consultation publique pour projeter à l'échelle européenne les taux d'adhésion ou d'opposition à l'ISDS, les résultats de cette consultation publique permettent néanmoins de voir comment se cristallisent les principaux arguments avancés par chacune des parties sur cet aspect du projet de TTIP.

\subsubsection{De l'ISDS au projet d'ICS}

Durant plusieurs mois, la société civile dénonce le fait que la Commission européenne ne prend pas en compte les enseignements de la consultation publique, puisqu'elle ne modifie pas sa position à l'égard de l'ISDS ${ }^{95}$. Cependant, en septembre 2015, la commissaire européenne au Commerce, C. Malmström, publie un projet d'adaptation de l'ISDS, qui s'y voit renommé système juridictionnel des investissements ( investment court system ", ICS) ${ }^{96}$. Ce projet vise à répondre au "manque de confiance de la part du public quant à l'équité et l'impartialité de l'ancien modèle de l'ISDS ». Avant d'en analyser les caractéristiques, il est important de noter dès à présent que, à l'heure actuelle, l'ICS n'est encore qu'un projet : il n'a pas encore été formalisé complètement au niveau européen (il doit encore être discuté au Conseil et au Parlement européen) et, de ce fait, n'a pas encore été soumis à l'avis du partenaire états-unien.

L'ICS apporte diverses nouveautés. À l'inverse de l'ISDS, cette juridiction serait établie sous la forme d'une cour permanente. Un système d'appel serait introduit, ce qui constitue aussi une innovation. En effet, l'ICS serait composé de deux cours (alors que l'ISDS n'en prévoyait qu'une seule) : le "tribunal de première instance » et le «tribunal d'appel ». Y siégeraient à nombre égal des juges européens, états-uniens et de pays tiers, pour un total de quinze magistrats dans le premier tribunal et de six dans le second. Des normes éthiques seraient imposées : les juges devraient se soumettre à un code de bonne conduite, percevoir une rémunération suffisante pour assurer une compétence et une indépendance sans faille, accepter de ne plus pouvoir siéger en tant qu'avocat dans ce genre de litiges. Afin d'éviter tout conflit d'intérêt, les juges seraient repartis de manière aléatoire sur les différentes affaires. De plus, tous les documents devraient être publiés pour assurer la transparence des procédures. Par ailleurs, certains éléments du mandat confié à la Commission européenne par le Conseil de l'Union européenne devraient être clarifiés

94 Ibidem, p. 15

95 Cf., par exemple, Centre national de coopération au développement (CNCD-11.11.11), «TTIP : la Commission est sourde », 14 janvier 2015, www.cncd.be.

96 C. MAlmStRÖM, « Proposing an Investment Court System », 16 septembre 2015, http://ec.europa.eu. 
dans le cadre des négociations visant à mettre en place l'ICS : il s'agirait d'expliciter, d'une part, le droit des États à réglementer et, d'autre part, les «standards de protection d'investissement » et les critères d'un " traitement juste et équitable » (afin de réduire le degré d'interprétation laissé aux juges).

La société civile accueille d'emblée positivement certaines nouveautés liées à l'ICS. En particulier, l'établissement d'une cour d'appel, le processus de sélection des juges et le développement d'un code éthique sont vus comme de nets progrès ${ }^{97}$. Cependant, de nombreux acteurs restent malgré tout très critiques vis-à-vis de l'ICS, qui n'est selon eux que le « zombie de l'ISDS » ${ }^{98}$. Tout d'abord, ce système maintiendrait toujours un biais favorable aux investisseurs, du fait qu'il serait unilatéral à l'instar de l'ISDS (il ne permet pas aux États d'attaquer les investisseurs) et que les investisseurs ne seraient soumis à aucun devoir allant avec leurs droits. Ensuite, même si le code éthique, la rémunération, ainsi que la répartition aléatoire des juges limiteraient les potentiels conflits d'intérêt, ces juges auraient tout de même intérêt à faire fonctionner un système pour lequel ils seraient rémunérés (et, concrètement, les investisseurs étant systématiquement les plaignants, il serait logique que les juges penchent en leur faveur). De plus, même s'il inclut une mention explicite du droit à légiférer des États, l'ICS ferait lui aussi office d'« épouvantail » pour les États qui voudraient adopter des législations susceptibles de nuire aux profits des investisseurs. Parallèlement, ce nouveau mécanisme n'empêcherait toujours pas des investisseurs d'attaquer des législations d'intérêt public. Enfin, le fait que les clauses du TTIP puissent être considérées légalement sur un même pied que le droit international (sous la "umbrella clause») pose d'autant plus question qu'un tel mécanisme a été rejeté par le Canada, qui le considérait comme trop dangereux, dans le cadre de la négociation du CETA ${ }^{99}$.

Au-delà de ces critiques, d'autres vont plus loin et remettent en cause l'institution même d'un tel mécanisme. L'existence de nombreux investissements qui ne sont pas couverts par l'ISDS remet en cause la nécessité d'un tel mécanisme, qui n'est d'ailleurs pas inclus dans tous les accords de libre-échange. De plus, dans le cas particulier des États-Unis, rien ne semble prouver qu'une telle clause soit nécessaire (alors que l'idée de l'inclure dans des accords avec des pays dont le système démocratique est moins fiable est davantage acceptée) ${ }^{100}$. Enfin, certains estiment que l'ICS serait illégal au regard des réglementations européennes. Ainsi, l'Association des magistrats allemands (Deutscher Richterbund) a publié une opinion dans laquelle elle questionne la base juridique sur laquelle l'Union européenne baserait l'établissement d'une telle cour ${ }^{101}$. D'autres arguent dans le même sens et mettent en évidence des incompatibilités entre l'ICS et la juridiction de la Cour européenne de justice ${ }^{102}$.

97 P. EBERHARDT, « The Zombie ISDS. Rebranded as ICS, Rights for Corporations to Sue States refuse to Die », s.d., CEO et al., mars 2016, p. 6, http://corporateeurope.org.

Ibidem.

99 Ibidem, p. 21

100 Seattle to Brussels Network (S2B), «ISDS: Courting Foreign Investors. Why the Commission’s Proposal for an "Investment Court System" Still Fails to Address the Key Problems of Foreign Investors' Privileges ", 29 septembre 2015, www.s2bnetwork.org.

101 Deutscher Richterbund, «Stellungnahme zur Errichtung eines Investitionsgerichts für TTIP. Vorschlag der Europäischen Kommission vom 16.09.2015 und 12.11.2015 », 4 février 2016, www.drb.de.

102 ClientEarth, «Legality of Investor-State Dispute Settlement (ISDS) under EU Law. Legal Study », 22 octobre 2015, http://documents.clientearth.org. 


\section{L'ÉTABLISSEMENT DE STANDARDS INTERNATIONAUX}

Le projet de TTIP vise à mettre en place des règles bilatérales entre l'Union européenne et les États-Unis. Toutefois, étant donné l'importance de ce projet et de ses deux protagonistes, de telles normes sont susceptibles de servir à l'avenir de référence dans l'établissement de standards mondiaux ${ }^{103}$. Ainsi, le mandat de négociation confié par le Conseil de l'Union européenne à la Commission européenne indique :

«L'objectif de l'accord est d'accroître le commerce et les investissements entre l'UE et les États-Unis en tirant parti du potentiel inexploité d'un véritable marché transatlantique, générant ainsi de nouvelles possibilités économiques en matière d'emploi et de croissance grâce à un accès accru au marché et à une meilleure compatibilité réglementaire et ouvrant la voie à des normes mondiales. »

Parmi les normes concernées, celles relatives aux droits de propriété intellectuelle sont particulièrement intéressantes pour le domaine agricole. En effet, le mandat donne l'instruction suivante :

«Les négociations viseront à garantir une protection et une reconnaissance accrues, grâce à l'accord, des indications géographiques de l'UE, d'une manière qui se fonde sur les [aspects des droits de propriété intellectuelle qui touchent au commerce] et les complète, en tenant compte du lien avec leur utilisation préalable sur le marché américain en vue de résoudre les conflits existants de manière satisfaisante. »

Les questions liées à la propriété intellectuelle ne sont pas sans liens avec la philosophie de production qu'adoptent l'Union européenne et les États-Unis dans le domaine agricole, dont on a indiqué que la seconde est marquée par un caractère industriel alors que la PAC s'est davantage attachée à maintenir une agriculture davantage familiale, multifonctionnelle et de qualité (cf. supra).

Dans le secteur de l'agriculture, la question de la propriété intellectuelle est directement liée à la reconnaissance des indications géographiques. Celles-ci renvoient à un produit fabriqué dans une zone géographique délimitée, qui jouit d'une certaine réputation et dont la qualité respecte certains critères précis ${ }^{104}$. Des exemples classiques sont le parmesan ou le champagne. Alors que les États-Unis sont très peu touchés par cette problématique (à l'exception, par exemple, du vin californien), les Européens, eux, y sont très attachés.

${ }^{103}$ HLWG, «Final Report », op. cit., p. 5.

104 J. GRUEFF, «Achieving a Successful Outcome for Agriculture in the EU-US Transatlantic Trade and Investment Partnership Agreement », op. cit., p. 19. 
Cela touche particulièrement les secteurs de la viande, du vin, de la bière et des produits laitiers, en particulier les fromages ${ }^{105}$.

Le problème principal pour les producteurs européens est que ces appellations sont utilisées de manière «semi générique » par les États-Unis ${ }^{106}$. Selon ces derniers, ces termes spécifiques servent à désigner des produits de la vie de tous les jours, qu'il ne serait donc pas nécessaire de protéger ${ }^{107}$. Néanmoins, il a été prouvé que l'utilisation de ces termes bénéficie aux rendements d'un produit qui porte une appellation de ce type ${ }^{108}$. Pour les Européens, les indications géographiques sont un aspect fondamental de leur agriculture, qu'il est indispensable de protéger pour plusieurs raisons. D'une part, ces appellations rappellent l'attachement que les producteurs européens ont aux traditions et à la culture de l'alimentation ; les partisans de cette position indiquent que cela fait même partie de l'identité des agriculteurs européens. D'autre part, économiquement, des appellations d'origine contrôlée permettent de compenser le manque de compétitivité de certains agriculteurs, particulièrement de petits cultivateurs ou éleveurs, qui privilégient la qualité à la quantité de production ${ }^{109}$. Pour garantir cela, il faut évidemment que ces indications géographiques soient reconnues et qu'il soit interdit aux producteurs états-uniens de produits similaires de les utiliser. En effet, tant qu'un accord n'est pas conclu, l'impact des indications géographiques est atténué par une sur-utilisation du terme. La volonté de nombreux agriculteurs européens est donc de maintenir une utilisation stricte des indications géographiques.

De ce point de vue, le TTIP pourrait, si les indications géographiques sont reconnues - ce à quoi les grandes firmes agro-alimentaires états-uniennes sont défavorables -, permettre aux producteurs européens de compenser leur manque de compétitivité dans certains secteurs par un niveau supérieur de qualité reconnu par ces appellations, et donc par un prix de vente supérieur. Dans le secteur laitier, ce serait particulièrement intéressant pour les producteurs européens, d'autant que cela empêcherait leurs homologues étatsuniens de produire certains fromages - ce qui, à nouveau, serait synonyme d'une demande accrue pour les produits européens ${ }^{110}$.

Cet aspect n'est toutefois pas encore tranché à l'heure actuelle: l'idée d'une telle reconnaissance des indications géographiques n'est encore ni acquise ni abandonnée. Néanmoins, la société civile européenne nourrit déjà quelques inquiétudes à ce sujet, à la lumière du résultat des négociations du CETA (le traité de libre-échange finalisé mais pas encore ratifié entre l'Union européenne et le Canada). En effet, alors que l'Union européenne revendiquait la reconnaissance de quelque 1000 appellations d'origine contrôlée, seules une centaine d'entres elles ont finalement été reconnues, dont aucune pour la Belgique ${ }^{111}$. Cependant, la Commission européenne affirme qu'elle «n'accepter[a] jamais de revoir à la baisse la protection dont bénéficient actuellement les IG

105

CEPII, «Risques et opportunités pour le secteur agroalimentaire européen liés à un possible accord commercial entre l'UE et les États-Unis », op. cit., p. 60.

106 Ibidem.

E. Trachtenberg, « A Transatlantic Partnership », op. cit., p. 15.

T. JosLing, S. TANGERMAnN, «Agriculture, Food and the TTIP », op. cit., p. 21.

E. Trachtenberg, « A Transatlantic Partnership », op. cit., p. 15.

10 J. GRUEFF, "Achieving a Successful Outcome for Agriculture in the EU-US Transatlantic Trade and Investment Partnership Agreement », op. cit., p. 21.

111 A. ZACHARIE, N. VAN NUFFEL, M. CERMAK, "Traité transatlantique (TTIP) : cartographie d'un partenariat controversé », op. cit., p. 35. 
[indications géographiques, équivalents des appellations d'origine contrôlée] en Europe. Les importations utilisant abusivement des appellations protégées ne pourront jamais se retrouver sur le marché de l'UE ${ }^{112}$. Cela semble suggérer que, même si les appellations d'origine contrôlée ne sont pas reconnues aux États-Unis, leur protection sera en tout cas maintenue en Europe.

112 Commission européenne, Direction générale Commerce, Au cœur du TTIP, op. cit., p. 45. 


\section{CONCLUSION}

Le projet de Partenariat transatlantique de commerce et d'investissement (Transatlantic Trade and Investment Partnership, TTIP) en cours de négociation entre l'Union européenne et les États-Unis soulève enthousiasme et oppositions, intérêt et critiques. En se concentrant sur les aspects agricoles du TTIP, ce Courrier hebdomadaire a tenté d'en éclairer les implications et les enjeux, notamment pour les consommateurs et pour les producteurs tant européens qu'états-uniens. Soulignons cependant que le propos développé ici s'est concentré sur le secteur de l'agriculture, à l'exclusion de tous les autres ; les observations et conclusions émises se limitent par conséquent à ce seul domaine. Par ailleurs, rappelons une fois de plus que le TTIP n'est à ce stade qu'un projet, toujours en cours de négociation.

Les différences de style dans l'approche politique de la question agricole par les deux protagonistes permettent de mettre en exergue les tensions que suscitent chacun des volets du projet et les opportunités qu'il comporte. Trois différences majeures ont été identifiées: la philosophie générale de production agricole, le degré de protection douanière et l'approche présidant à l'établissement des normes.

Le premier volet des négociations, relatif à l'amélioration de l'accès au futur marché commun, concerne les questions d'ordre tarifaire. La levée de toute barrière de cette nature bénéficierait proportionnellement plus aux États-Unis, les exportations de ceux-ci vers les États membres de l'Union européenne étant jusqu'à présent freinées par des tarifs importants dans des secteurs clés comme ceux du sucre, de la viande ou des produits laitiers. En outre, le caractère industriel prononcé de la production agricole états-unienne accroîtrait les avantages de ces agriculteurs en cas de libéralisation. Pour leur part, mais dans une moindre mesure, il est vrai, les Européens pourraient bénéficier de cette levée dans des secteurs états-uniens protégés comme celui des produits laitiers. Le récent rapport du Ministère de l'Agriculture des États-Unis (United States Department of Agriculture, USDA) confirme ces observations. Il prévoit, par exemple, une augmentation de $685 \%$ de l'exportation de bœuf états-unien vers l'Europe. Dans l'autre sens, les plus grands gains seraient réalisés dans le secteur du fromage.

Il faut cependant relativiser l'impact isolé d'une levée des barrières tarifaires, qui n'influencerait que dans une moindre mesure les bénéfices du TTIP. Toutes les études sur lesquelles s'appuie la présente analyse s'accordent pour estimer que, si seules les barrières tarifaires sont levées, le gain d'exportations bilatérales sera seulement d'entre 25 et $50 \%$ de ce qu'il pourrait être dans un scénario incluant également la levée ou l'harmonisation des barrières non tarifaires. Ce second aspect revêt donc une importance cruciale dans les négociations. L'intérêt qu'y portent la société civile européenne et la 
Commission européenne en témoigne, de même que l'existence ou la préparation de diverses études relatives à l'impact potentiel du futur TTIP si celui-ci est adopté. Ici, le facteur déterminant pour comprendre les enjeux est la différence d'approche entre l'Union européenne et les États-Unis pour établir des normes, la première se basant à la fois sur le principe de précaution et sur un contrôle « de la ferme à la table » et la seconde reposant « sur la science ». Ce critère pourrait avoir un impact décisif en ce qui concerne une éventuelle convergence réglementaire. D'une part, si un système de reconnaissance mutuelle est mis en place, les producteurs européens pourraient être victimes de concurrence déloyale tandis que les consommateurs européens pourraient se sentir mis en danger en termes de santé publique. D'autre part, si le TTIP mène à une harmonisation, le risque principal pour les Européens est un alignement des normes sur les standards états-uniens, puisque ceux-ci sont établis selon les critères de l'Organisation mondiale du commerce (OMC). En outre, l'existence d'un système tel que le système juridictionnel des investissements («investment court system», ICS) pourrait rendre plus facilement attaquable toute mesure publique ayant pour conséquence incidente une limitation du profit des investisseurs, quel que soit par ailleurs son objet réel. Cette hypothèse est néanmoins réfutée à de nombreuses reprises par la Commission européenne et en particulier sa représentante dans le projet, la commissaire au Commerce Cecilia Malmström.

Pour sa part, la levée des obstacles techniques au commerce, qui ne concerne pas directement des mesures sanitaires ou phytosanitaires, pourrait être bénéfique aux agriculteurs européens actifs dans le secteur laitier. De même, la reconnaissance potentielle des indications géographiques apparaît comme le point le plus intéressant pour les éleveurs et cultivateurs européens, puisqu'elle permettrait le maintien d'un prix supérieur pour certains de leurs produits, en raison d'une différence de qualité. Dans ce cas-ci, cependant limité, la différence de philosophie de production pourrait donc avantager les producteurs européens.

Quelles que soient les nuances que l'on peut apporter, le rapport de l'USDA montre tout de même que, en termes absolus, les États-Unis profiteraient largement plus que l'Union européenne de la mise en œuvre du TTIP dans le domaine agricole, et ce quel que soit le scénario envisagé. Certes, le choix des hypothèses sur lesquelles repose cette recherche prospective a sans doute été politiquement orienté de manière à obtenir des résultats positifs pour les États-Unis. Mais il n'empêche que ce rapport pose question. À la lecture des chiffres qu'il fournit et dans les conditions qu'il envisage, la volonté de l'Union européenne d'inclure le secteur agricole dans les négociations actuelles semble difficilement compréhensible, puisque tel n'est manifestement pas l'intérêt des Européens. Peut-être cela relève-t-il d'un hypothétique choix européen d'accepter d'enregistrer des pertes dans le secteur agricole afin de récolter des bénéfices, supérieurs, dans d'autres secteurs.

À travers ce Courrier hebdomadaire, on voit que les approches des deux partenaires potentiels en termes de politique agricole restent fort différentes. Cela peut engendrer des pertes ou des bénéfices pour les différents acteurs. Les deux premiers aspects envisagés rejoignent l'approche états-unienne, sur laquelle les Européens seraient probablement contraints de s'aligner. Cela aurait pour ces derniers un coût indéniable. Le volet relatif aux normes sanitaires et phytosanitaires semble être le plus problématique pour l'Union européenne, vu son approche peu en phase avec celle de l'OMC. En revanche, certaines 
opportunités pourraient se présenter aux Européens en raison de la levée des barrières tarifaires et des obstacles techniques au commerce, et si l'approche qualitative des produits européens est reconnue à travers la reconnaissance des indications géographiques.

Surtout, ce qui ressort de notre analyse, et qui constitue sûrement l'un des problèmes les plus fondamentaux liés au projet de TTIP, est l'ambivalence du mandat confié à la Commission européenne par le Conseil de l'Union européenne. En effet, pour les trois volets considérés, les directives de négociation données par les États membres sont sujettes à interprétation. Ce manque de clarté nuit à l'organisation d'un débat réellement constructif dans la société civile. En effet, un certain nombre des désaccords qui existent entre les partisans du TTIP et ses opposants apparaissent basés sur le fait que chacun se réfère à des éléments différents du mandat. En outre, combiné au secret qui entoure le contenu concret du traité en négociation, ce manque de clarté semble nourrir les inquiétudes des opposants, amenés à développer des thèses parfois hypothétiques sur la base d'exemples extérieurs. Certes, depuis l'entrée en fonction de C. Malmström, la Commission a développé une approche qui se veut plus transparente et pédagogique en matière de communication relative au TTIP. Néanmoins, au vu des vives oppositions qui continuent de viser le projet de TTIP, cela ne semble pas suffisant pour convaincre l'ensemble de la société civile du bien-fondé de celui-ci.

Quoi qu'il en soit, l'intérêt pour les consommateurs et pour les agriculteurs européens dépendra surtout du choix que l'Union européenne posera dans la suite des négociations relatives au projet de TTIP : pour autant qu'elle choisisse de maintenir le secteur agricole dans le périmètre des tractations, à quel point défendra-t-elle la protection de l'environnement, des consommateurs et des petits producteurs - autant d'éléments que le TTIP, dans son état actuel, ne semble pas forcément garantir? 


\section{ANNEXE}

\section{Mandat de négociation délivré à la Commission européenne par le Conseil des affaires étrangères (Questions commerciales) le 14 juin 2013 concernant le projet de Partenariat transatlantique de commerce et d'investissement entre l'Union européenne et les États-Unis d'Amérique}

Directives de négociation concernant un accord global sur le commerce et l'investissement, appelé Partenariat transatlantique de commerce et d'investissement, entre l'Union européenne et les États-Unis d'Amérique

\section{Nature et portée de l'accord}

L'accord contiendra exclusivement des dispositions relatives aux questions commerciales et liées au commerce qui sont applicables entre les parties. L'accord devrait confirmer que le partenariat transatlantique de commerce et d'investissement est fondé sur des valeurs communes, notamment la protection et la promotion des droits de l'homme et de la sécurité internationale.

L'accord devra être ambitieux, complet, équilibré et parfaitement conforme aux règles de l'Organisation mondiale du commerce (OMC) et aux obligations imposées par celle-ci.

L'accord devra permettre la libéralisation réciproque des échanges de biens et de services et prévoir des règles applicables aux questions liées au commerce, avec un niveau d'ambition élevé, dépassant celui des engagements pris précédemment dans le cadre de l'OMC.

Les obligations de l'accord seront obligatoires à tous les niveaux de gouvernement.

L'accord sera composé de trois volets principaux : a) accès au marché, b) questions réglementaires et obstacles non tarifaires, et c) règles. Ces trois volets seront négociés en parallèle et feront partie d'un engagement unique garantissant un résultat équilibré entre la suppression des droits, la suppression des obstacles réglementaires superflus et une amélioration des règles, conduisant à des résultats substantiels dans chacun de ces volets et à une ouverture effective des marchés de chacune des parties.

\section{Préambule et principes généraux}

Le préambule rappellera que le partenariat avec les États-Unis repose sur des valeurs et principes communs qui sont cohérents avec les principes et objectifs de l'action extérieure de l'Union. Il évoquera notamment : 
- les valeurs communes dans des domaines tels que les droits de l'homme, les libertés fondamentales, la démocratie et la primauté du droit,

- l'engagement des parties en faveur du développement durable et la contribution des échanges internationaux au développement durable sur le plan économique, social et environnemental, y compris en matière de développement économique, de plein emploi productif et de conditions de travail décentes pour tous, ainsi que la protection et la préservation de l'environnement et des ressources naturelles,

- l'engagement des parties à conclure un accord qui respecte pleinement leurs droits et obligations dans le cadre de l'OMC et soutient le système commercial multilatéral,

- le droit des parties à prendre les mesures nécessaires pour atteindre des objectifs de politique publique légitimes, en fonction du niveau de protection de la santé, de la sécurité, des travailleurs, des consommateurs, de l'environnement et de la promotion de la diversité culturelle, telle qu'elle est énoncée dans la convention de l'UNESCO sur la protection et la promotion de la diversité des expressions culturelles, qu'elles jugent approprié,

- l'objectif commun des parties de prendre en compte les problèmes particuliers rencontrés par les petites et moyennes entreprises dans le cadre de leur contribution au développement du commerce et des investissements,

- l'engagement des parties de communiquer avec toutes les parties intéressées, y compris les organisations du secteur privé et de la société civile.

\section{Objectifs}

L'objectif de l'accord est d'accroître le commerce et les investissements entre l'UE et les États-Unis en tirant parti du potentiel inexploité d'un véritable marché transatlantique, générant ainsi de nouvelles possibilités économiques en matière d'emploi et de croissance grâce à un accès accru au marché et à une meilleure compatibilité réglementaire et ouvrant la voie à des normes mondiales.

L'accord devrait reconnaître que le développement durable est l'un des objectifs principaux des parties et que ces dernières s'efforceront de garantir et de faciliter le respect des normes et accords internationaux en matière d'environnement et de travail, tout en favorisant de hauts niveaux de protection de l'environnement, des travailleurs et des consommateurs, conformément à l'acquis de l'UE et à la législation des États membres. L'accord devrait établir que les parties ne favoriseront pas les échanges ou les investissements directs étrangers en réduisant la portée de la législation et des normes internes en matière d'environnement, d'emploi ou de santé et sécurité au travail, ou en assouplissant les normes fondamentales du travail ou les politiques et la législation visant à protéger et à promouvoir la diversité culturelle.

L'accord ne devra contenir aucune disposition risquant de porter atteinte à la diversité culturelle et linguistique de l'Union ou de ses États membres, notamment dans le secteur culturel, ni d'empêcher l'Union et ses États membres de conserver les politiques et mesures en vigueur visant à soutenir le secteur culturel, compte tenu de son statut spécial au sein de l'UE et de ses États membres. L'accord ne portera pas atteinte à la capacité de l'Union et de ses États membres à mettre en ouvre des mesures visant à tenir compte des évolutions de ce secteur en particulier dans l'environnement numérique. 


\section{ACCÈS AU MARCHÉ}

\section{Échanges de biens}

\section{Droits de douane et autres exigences à l'importation et à l'exportation}

L'accord aura pour but de supprimer l'ensemble des droits sur les échanges bilatéraux. L'objectif commun sera la suppression d'un grand nombre de droits de douane au moment de l'entrée en vigueur de l'accord, suivie de la suppression progressive de tous les droits de douane, à l'exception des plus sensibles, à brève échéance. Lors des négociations, les deux parties examineront des options pour le traitement des produits les plus sensibles, y compris les contingents tarifaires. Tous les droits de douane, taxes, prélèvements ou redevances à l'exportation ainsi que les restrictions quantitatives ou les exigences en matière d'autorisation concernant les exportations vers l'autre partie qui ne sont pas justifiés par des exceptions dans le cadre de l'accord seront supprimés au moment de l'entrée en vigueur de l'accord. Les négociations s'attacheront à répondre aux préoccupations concernant les obstacles restants au commerce des biens à double usage qui portent atteinte à l'intégrité du marché unique.

\section{Règles d'origine}

Les négociations viseront à concilier les approches de l'UE et des États-Unis en matière de règles d'origine, d'une manière qui facilitera les échanges entre les parties et tiendra compte des règles d'origine de l'UE et des intérêts des producteurs européens. Elles devraient aussi avoir pour objet de garantir le traitement approprié des erreurs administratives. À la suite d'une présentation par la Commission d'une analyse de ses éventuelles conséquences économiques et dans le cadre d'une consultation préalable avec le comité de la politique commerciale, les possibilités de cumul avec des pays voisins ayant conclu des accords de libre-échange (ALE) à la fois avec l'UE et avec les États-Unis seront prises en considération.

\section{Exceptions générales}

L'accord inclura une clause d'exception générale fondée sur les articles XX et XXI du GATT.

\section{Mesures antidumping et compensatoires}

L'accord devrait comporter une clause sur les mesures antidumping et compensatoires, reconnaissant que chacune des parties peut prendre des mesures appropriées contre le dumping et/ou les subventions passibles de droits compensateurs conformément à l'accord de l'OMC sur la mise en œuvre de l'article VI de l'Accord général sur les tarifs douaniers et le commerce de 1994 ou à l'accord de l'OMC relatif aux subventions et aux mesures compensatoires. L'accord devrait instituer un dialogue régulier sur les questions de défense commerciale.

\section{Mesures de sauvegarde}

Afin de maximiser les engagements en matière de libéralisation, l'accord devrait contenir une clause de sauvegarde bilatérale permettant à chaque partie de retirer, partiellement ou intégralement, le bénéfice des préférences si une augmentation des importations d'un produit provenant de l'autre partie cause ou menace de causer un préjudice grave à sa branche de production intérieure. 


\section{Commerce de services et établissement}

Les négociations concernant le commerce de services auront pour objet le maintien du niveau de libéralisation autonome des deux parties au niveau le plus élevé de libéralisation prévu par les ALE en vigueur, conformément à l'article V de l'AGCS, couvrant substantiellement tous les secteurs et tous les modes de prestation, tout en obtenant un nouvel accès au marché grâce à la suppression des obstacles restants, qui existent de longue date, en tenant dûment compte du caractère sensible de certains secteurs. De plus, les États-Unis et l'UE incluront des engagements contraignants destinés à assurer la transparence, l'impartialité et la régularité de traitement en ce qui concerne les exigences et les procédures en matière de licences et de qualifications, et à renforcer les disciplines réglementaires figurant dans les ALE actuels des États-Unis et de l'UE.

Les parties devraient convenir d'accorder un traitement non moins favorable pour l'établissement sur leur territoire de sociétés, de filiales ou de succursales de l'autre partie que celui accordé à leurs propres sociétés, filiales ou succursales, en tenant dûment compte du caractère sensible de certains secteurs spécifiques.

L'accord devrait mettre en place un cadre visant à faciliter la reconnaissance mutuelle des qualifications professionnelles.

L'accord ne fera pas obstacle à l'application d'exceptions concernant la prestation de services qui sont compatibles avec les règles de l'OMC applicables en la matière (articles XIV et XIVbis de l'AGCS). La Commission devrait aussi veiller à ce qu'aucune disposition de l'accord ne fasse obstacle à l'application, par les parties, de leurs lois, réglementations et prescriptions nationales concernant l'admission et le séjour, à condition que n'en soient pas réduits à néant ou compromis les avantages découlant de l'accord. Les lois, réglementations et prescriptions de l'UE et des États membres concernant le travail et les conditions de travail continuent de s'appliquer.

La qualité élevée des services publics européens devrait être préservée conformément au TFUE et, en particulier, au protocole $\mathrm{n}^{\circ} 26$ sur les services d'intérêt général et compte tenu des engagements de l'UE en la matière, notamment dans le cadre de l'AGCS.

Les services fournis dans l'exercice du pouvoir gouvernemental définis à l'article $1^{\text {er }}$, paragraphe 3, de l'AGCS seront exclus des présentes négociations.

Les services audiovisuels ne seront pas couverts par le présent chapitre.

\section{Protection des investissements}

Les négociations relatives aux investissements porteront sur des dispositions concernant la libéralisation et la protection des investissements, y compris les domaines de compétence mixte comme les aspects relatifs aux investissements de portefeuille, à la propriété et à l'expropriation, sur la base du niveau le plus élevé de libéralisation et des normes les plus élevées de protection que les deux parties ont négociés à ce jour. Après consultation préalable des États membres et conformément aux traités UE, la protection des investissements et le règlement des différends entre les investisseurs et l'État (RDIE) seront inclus si une solution satisfaisante qui répond aux intérêts de l'UE concernant les questions couvertes par le point 23 est trouvée. Cette question sera en outre examinée dans la perspective de l'équilibre final de l'accord. 
En ce qui concerne la protection des investissements, les dispositions de l'accord devraient :

- garantir le niveau de protection et de sécurité juridique le plus élevé possible pour les investisseurs européens aux États-Unis,

- mettre en avant les normes européennes en matière de protection, ce qui devrait permettre de renforcer l'attractivité de l'Europe en tant que destination d'investissements étrangers,

- instaurer des conditions de concurrence équitables pour les investisseurs aux États-Unis et dans l'UE,

- faire fond sur l'expérience des États membres et leurs pratiques exemplaires en matière d'accords bilatéraux d'investissement avec des pays tiers,

- $\quad$ être sans préjudice du droit de l'UE et des États membres d'adopter et d'appliquer, conformément à leurs compétences respectives, les mesures nécessaires pour poursuivre de manière non discriminatoire des objectifs légitimes de politique publique, par exemple en ce qui concerne le domaine social, l'environnement, la sécurité, la stabilité du système financier, la santé et la sécurité publiques. L'accord devrait respecter les politiques appliquées par l'UE et par ses États membres pour privilégier et protéger la diversité culturelle.

Champ d'application : le chapitre de l'accord portant sur la protection des investissements devrait couvrir un large éventail d'investisseurs et leurs investissements, y compris les droits de propriété intellectuelle, que l'investissement soit effectué avant ou après l'entrée en vigueur de l'accord.

Normes de traitement : les négociations devraient viser à inclure en particulier, mais pas exclusivement, les normes de traitement et règles suivantes :

a) un traitement juste et équitable, comportant l'interdiction de mesures déraisonnables, arbitraires ou discriminatoires ;

b) un traitement national ;

c) un traitement de la nation la plus favorisée ;

d) la protection contre l'expropriation directe et indirecte, y compris le droit à une compensation rapide, adéquate et efficace ;

e) la pleine protection et la pleine sécurité des investisseurs et des investissements ;

f) d'autres dispositions de protection efficaces, par exemple une " clause de protection »;

g) le libre transfert des fonds par les investisseurs (capitaux et paiements);

h) des règles en matière de subrogation.

Application : l'accord devrait viser à créer un mécanisme efficace et moderne de règlement des différends entre les investisseurs et l'État qui garantisse la transparence, l'indépendance des arbitres et la prévisibilité de l'accord, y compris par la possibilité de lier les parties pour ce qui est de leur interprétation de l'accord. Le règlement des différends entre États devrait être inclus dans ce mécanisme, mais il ne devrait pas porter atteinte au droit des investisseurs de recourir à des mécanismes de règlement des différends entre les investisseurs et l'État. L'accord devrait offrir aux investisseurs une palette de structures d'arbitrage aussi large que celle qui existe dans le cadre des traités bilatéraux d'investissement entre les États membres. Le mécanisme de règlement des différends entre les investisseurs et l'État devrait contenir des mesures de sauvegarde contre les réclamations 
manifestement injustifiées ou abusives. Il conviendra d'envisager la création d'un mécanisme d'appel applicable au règlement des différends entre les investisseurs et l'État au titre de l'accord, et d'étudier la relation qu'il convient d'établir entre le RDIE et les voies de recours internes.

Lien avec les autres parties de l'accord : les dispositions sur la protection des investissements devraient être sans rapport avec les engagements en matière d'accès au marché concernant les investissements pris dans d'autres parties de l'accord. Les RDIE ne s'appliqueront pas aux dispositions concernant l'accès au marché. Ces engagements en matière d'accès au marché peuvent inclure, si nécessaire, des règles interdisant les exigences de résultats.

Toutes les autorités et entités sous-centrales (comme les États ou les municipalités) devraient se conformer effectivement au chapitre relatif à la protection des investissements de cet accord.

\section{Marchés publics}

L'accord devra être le plus ambitieux possible et compléter les résultats des négociations relatives à la révision de l'accord sur les marchés publics en ce qui concerne son champ d'application (entités contractantes, secteurs, valeurs de seuil et contrats de services, notamment pour les travaux publics de construction). L'accord visera à accroître l'accès mutuel aux marchés publics à tous les niveaux administratifs (national, régional et local) et dans le domaine des services publics, afin de s'appliquer aux activités pertinentes des entreprises actives dans ce domaine, en garantissant un traitement non moins favorable que celui qui est accordé aux fournisseurs établis sur le territoire de la partie concernée. L'accord comprendra également des règles et disciplines permettant de lutter contre les obstacles ayant des conséquences négatives pour les marchés publics de chaque partie, y compris les exigences de contenu local ou de production locale, en particulier les dispositions "achetez américain », et celles qui s'appliquent aux procédures d'appel d'offres, aux spécifications techniques, aux procédures de recours et aux exceptions existantes, $y$ compris pour les petites et moyennes entreprises, en vue d'accroître l'accès au marché et, s'il y a lieu, de rationaliser, simplifier et rendre plus transparentes les procédures.

\section{QUESTIONS RÉGLEMENTAIRES ET OBSTACLES NON TARIFAIRES}

L'accord visera à supprimer, par des mécanismes efficaces, les obstacles superflus au commerce et aux investissements, y compris les obstacles non tarifaires en vigueur, en parvenant à un niveau ambitieux de compatibilité réglementaire pour les biens et les services, y compris par la reconnaissance mutuelle, l'harmonisation et le renforcement de la coopération entre régulateurs.

La compatibilité réglementaire ne fera pas obstacle au droit de réglementer en fonction du niveau de protection de la santé, de la sécurité, des consommateurs, des travailleurs, de l'environnement et de la diversité culturelle que chaque partie juge approprié, ou de manière à atteindre des objectifs réglementaires légitimes, et sera conforme aux objectifs énoncés au point 8 . À cet effet, l'accord comprendra des dispositions portant sur les éléments ci-après. 


\section{- Mesures sanitaires et phytosanitaires (mesures SPS)}

En ce qui concerne les mesures SPS, les négociations devront suivre les directives de négociation adoptées par le Conseil le 20 février 1995 (document n 4976/95 du Conseil). Les parties élaboreront des dispositions reposant sur l'accord de l'OMC sur les mesures sanitaires et phytosanitaires ainsi que sur les dispositions de l'accord vétérinaire en vigueur, introduiront des disciplines en matière de protection phytosanitaire et institueront un forum bilatéral pour l'amélioration du dialogue et de la coopération sur les questions liées aux mesures SPS. Dans les domaines auxquels s'applique l'accord vétérinaire actuel entre l'UE et les États-Unis, les dispositions correspondantes devraient être considérées comme le point de départ des négociations. Les dispositions du chapitre consacré aux mesures SPS développeront les principes essentiels de l'accord de l'OMC sur les mesures SPS, y compris l'exigence en vertu de laquelle les mesures SPS de chaque partie doivent reposer sur une base scientifique et sur des normes internationales ou sur des évaluations des risques de nature scientifique, tout en reconnaissant le droit des parties à apprécier et gérer les risques conformément au niveau de protection que chaque partie juge approprié, en particulier lorsque les preuves scientifiques pertinentes sont insuffisantes, mais doivent n'être appliquées que dans la mesure nécessaire pour protéger la santé et la vie des personnes et des animaux ou préserver les végétaux, et doivent être élaborées de manière transparente, sans retard indu. L'accord devrait aussi viser à établir entre les parties des mécanismes de coopération portant, entre autres, sur l'équivalence des conditions de bien-être animal.

L'accord devrait viser à parvenir à une totale transparence en ce qui concerne les mesures sanitaires et phytosanitaires applicables au commerce, notamment à établir des dispositions pour la reconnaissance de l'équivalence, pour la mise en ouvre du pré-listing des établissements de production alimentaire, pour empêcher la mise en ouvre du prédédouanement, pour la reconnaissance du statut sanitaire indemne des parties vis-à-vis des maladies et des parasites et concernant le principe de la régionalisation des maladies animales et des maladies des plantes.

\section{- Réglementations techniques, normes et procédures d'évaluation de la conformité}

S'appuyant sur les engagements qu'elles ont pris au titre de l'accord de l'OMC sur les obstacles techniques au commerce (accord OTC), les parties devront également développer et compléter ces dispositions, afin de faciliter l'accès de chacune aux marchés de l'autre, et elles mettront en place un mécanisme permettant d'améliorer le dialogue et la coopération en ce qui concerne le traitement des questions bilatérales en la matière. Les dispositions ainsi élaborées devraient avoir pour objet d'accroître l'ouverture, la transparence et la convergence des méthodes et exigences réglementaires et des processus connexes d'élaboration de normes, également en vue de l'adoption de normes internationales pertinentes, ainsi que, notamment, de réduire les exigences redondantes et pesantes en matière d'essais et de certification, de favoriser la confiance de chaque partie dans les organismes d'évaluation de la conformité de l'autre et d'accroître la coopération en matière d'évaluation de la conformité et de normalisation d'une manière générale. Il convient aussi de se pencher sur les dispositions relatives à l'étiquetage et les moyens d'éviter les informations de nature à induire en erreur les consommateurs. 


\section{- Cohérence réglementaire}

L'accord comprendra des disciplines transversales concernant la cohérence réglementaire et la transparence pour l'élaboration et l'application de réglementations efficaces, économiquement performantes et plus compatibles en matière de biens et de services, y compris des consultations à un stade précoce sur des réglementations importantes, le recours à des analyses d'impact, des évaluations, des réexamens périodiques des mesures réglementaires en vigueur et l'application de bonnes pratiques réglementaires.

\section{- Dispositions sectorielles}

L'accord comportera des dispositions ou des annexes contenant des engagements ou des étapes supplémentaires visant à favoriser la compatibilité réglementaire pour certains biens et services particuliers définis d'un commun accord, en vue de réduire les coûts découlant des différences de réglementation dans certains secteurs, y compris la prise en considération de démarches relatives à l'harmonisation des réglementations, l'équivalence ou la reconnaissance mutuelle, selon le cas. Il devrait notamment s'agir de dispositions de fond et de procédures spécifiques dans des secteurs présentant un intérêt majeur pour l'économie transatlantique, à savoir, entre autres, l'automobile, les produits chimiques, les produits pharmaceutiques et les autres secteurs de la santé, les technologies de l'information et de la communication ainsi que les services financiers, garantissant la suppression des obstacles non tarifaires existants, évitant la mise en place de nouveaux obstacles de ce type et permettant un accès au marché plus important que celui qui sera garanti par les règles horizontales de l'accord. En ce qui concerne les services financiers, les négociations devraient également avoir pour objectif des cadres communs pour la coopération prudentielle.

L'accord devra également comprendre un cadre permettant de déceler les possibilités et d'orienter les travaux ultérieurs sur les questions de réglementation, y compris des dispositions fournissant une base institutionnelle pour intégrer l'issue des discussions réglementaires ultérieures dans l'accord général.

L'accord devrait être contraignant pour tous les régulateurs et toutes les autres autorités compétentes des deux parties.

\section{RÈGLES}

\section{Droits de propriété intellectuelle}

L'accord devra traiter les questions liées aux droits de propriété. L'accord reflétera la valeur élevée que revêt pour les deux parties la protection de la propriété intellectuelle et se fondera sur le dialogue UE- États-Unis existant dans ce domaine.

Les négociations devraient, en particulier, porter sur les domaines qui revêtent le plus d'importance pour l'échange de biens et de services dont le contenu est protégé par des droits de propriété intellectuelle, de manière à encourager l'innovation. Les négociations viseront à garantir une protection et une reconnaissance accrues, grâce à l'accord, des indications géographiques de l'UE, d'une manière qui se fonde sur les ADPIC et les complète, en tenant compte du lien avec leur utilisation préalable sur le marché américain en vue de résoudre les conflits existants de manière satisfaisante. Après consultation 
préalable du comité de la politique commerciale, les autres questions relatives aux droits de propriété intellectuelle seront examinées au cours des négociations.

L'accord ne comportera pas de dispositions sur les sanctions pénales

\section{Commerce et développement durable}

L'accord comprendra des engagements des deux parties en ce qui concerne les aspects du commerce et du développement durable touchant au travail et à l'environnement. Des mesures destinées à faciliter et encourager le commerce de biens respectueux de l'environnement et à faible teneur en carbone, de biens, services et technologies économes en énergie et en ressources, y compris par les marchés publics écologiques, ainsi qu'à permettre aux consommateurs de prendre leurs décisions d'achat en connaissance de cause seront examinées. L'accord contiendra également des dispositions encourageant l'adhésion aux normes et accords internationalement reconnus dans les domaines du travail et de l'environnement, ainsi que leur mise en œuvre effective, comme condition indispensable au développement durable.

L'accord prévoira des mécanismes visant à soutenir les actions en faveur du travail décent grâce à la mise en œuvre effective, sur le plan intérieur, des normes fondamentales du travail fixées par l'Organisation internationale du travail (OIT), telles que définies dans la déclaration de l'OIT de 1998 relative aux principes et droits fondamentaux au travail et dans les accords multilatéraux sur l'environnement se rapportant à ce domaine, et à renforcer la coopération sur les aspects commerciaux du développement durable. Il conviendra également de souligner qu'il importe d'appliquer et de faire respecter la législation interne dans les domaines du travail et de l'environnement. L'accord devrait aussi comporter des dispositions invitant au respect des normes internationalement reconnues en matière de responsabilité sociale des entreprises et appeler à la conservation et à la gestion durable des ressources naturelles, ainsi qu'à la promotion du commerce de ressources naturelles durables obtenues légalement, telles que le bois, la faune sauvage ou les produits de la pêche. L'accord prévoira le suivi de la mise en œuvre de ces dispositions par un mécanisme faisant appel à la participation de la société civile, ainsi qu'un mécanisme de règlement des différends.

Les incidences économiques, sociales et environnementales seront examinées au moyen d'une évaluation indépendante, associant la société civile, de l'impact sur le développement durable qui sera réalisée parallèlement aux négociations et sera achevée avant que l'accord ne soit paraphé. L'évaluation de l'impact sur le développement durable visera à préciser les effets probables de l'accord sur le développement durable et à proposer des mesures (dans des domaines commerciaux et non commerciaux) pour augmenter autant que possible les avantages de l'accord et éviter et réduire au minimum les incidences négatives éventuelles. La Commission veillera à ce que l'évaluation de l'impact sur le développement durable s'effectue dans le cadre d'un dialogue régulier avec l'ensemble des acteurs concernés de la société civile. Au cours des négociations, la Commission entretiendra également un dialogue régulier avec tous les acteurs concernés de la société civile.

\section{Questions douanières et facilitation des échanges}

L'accord devra contenir des dispositions destinées à faciliter les échanges entre les parties tout en garantissant des contrôles efficaces et des mesures antifraude. À cet effet, il devra 
notamment inclure des engagements concernant les règles, les exigences, les formalités et les procédures des parties en matière d'importation, d'exportation et de transit qui se caractériseront par un niveau d'ambition élevé et iront au-delà des engagements négociés au sein de l'OMC. Ces dispositions devraient promouvoir la modernisation et la simplification des règles et procédures, une documentation standardisée, la transparence, la reconnaissance mutuelle des normes et la coopération entre les autorités douanières.

\section{Accords commerciaux sectoriels}

L'accord devrait, le cas échéant, revoir, développer et compléter les accords commerciaux sectoriels existants, tels que l'accord entre la Communauté européenne et les ÉtatsUnis concernant le commerce du vin, en particulier en ce qui concerne la négociation des conditions dans le cadre de l'annexe II de l'accord de 2005, l'accord de reconnaissance mutuelle entre la Communauté européenne et les États-Unis et l'accord de coopération douanière et d'assistance mutuelle en matière douanière entre la Communauté européenne et les États-Unis d'Amérique.

\section{Commerce et concurrence}

Il faudrait viser à ce que l'accord contienne des dispositions dans le domaine de la politique de la concurrence, y compris en ce qui concerne les ententes, les concentrations et les aides d'État. Il devrait en outre traiter les monopoles d'États, les entreprises publiques et les entreprises bénéficiant de droits spéciaux ou exclusifs.

\section{Aspects de l'énergie et des matières premières qui touchent au commerce}

L'accord contiendra des dispositions concernant les aspects de l'énergie et des matières premières qui touchent au commerce et à l'investissement. Les négociations devraient viser à garantir un environnement économique ouvert, transparent et prévisible en matière d'énergie et un accès illimité et durable aux matières premières.

\section{Petites et moyennes entreprises}

L'accord contiendra des dispositions concernant les aspects des petites et moyennes entreprises qui touchent au commerce.

\section{Mouvements de capitaux et paiements}

L'accord contiendra des dispositions concernant la pleine libéralisation des paiements courants et des mouvements de capitaux et inclura une clause de statu quo. Il comportera des dispositions relatives aux exceptions (par exemple, en cas de graves difficultés ayant une incidence sur la politique monétaire et de change, à des fins de contrôle prudentiel ou dans le domaine de la fiscalité), qui seront conformes aux dispositions du traité UE relatives à la libre circulation des capitaux. Les négociations devront tenir compte des aspects sensibles de la libéralisation des mouvements de capitaux n'ayant pas de rapport avec les investissements directs.

\section{Transparence}

L'accord traitera les questions liées à la transparence. À cet effet, il inclura des dispositions concernant : 
- l'engagement de consulter les parties prenantes avant d'introduire des mesures ayant des conséquences sur le commerce et l'investissement,

- la publication des règles et mesures générales ayant des répercussions sur les échanges internationaux de biens et de services, ainsi que sur les investissements correspondants,

- la transparence dans la mise en œuvre de mesures ayant des répercussions sur les échanges internationaux de biens et de services, ainsi que sur les investissements correspondants.

Aucune disposition de l'accord ne devrait porter atteinte aux législations de l'UE ou des États membres concernant l'accès du public aux documents officiels.

\section{Autres domaines soumis à des règles}

Après analyse de la Commission, dans le cadre d'une consultation préalable avec le comité de la politique commerciale et conformément aux traités UE, l'accord peut contenir des dispositions afférentes à d'autres domaines liés aux relations commerciales et économiques si, au cours des négociations, un intérêt mutuel se dégage dans ce sens.

\section{Cadre institutionnel et dispositions finales}

\section{Cadre institutionnel}

L'accord mettra en place une structure institutionnelle permettant d'assurer un suivi efficace des engagements découlant de l'accord, ainsi que de favoriser la réalisation progressive de la compatibilité entre les régimes de réglementation.

La Commission, dans un esprit de transparence, rendra régulièrement compte de l'évolution des négociations au comité de la politique commerciale. Elle pourra, conformément aux traités, présenter des recommandations au Conseil sur d'éventuelles directives de négociation supplémentaires concernant n'importe quelle question, selon les mêmes procédures d'adoption, y compris les règles de vote, que pour le présent mandat.

\section{Règlement des différends}

L'accord comprendra un mécanisme approprié de règlement des différends qui garantira que les parties respectent les règles dont elles sont convenues.

L'accord devrait comporter des dispositions offrant des solutions rapides en matière de résolution des problèmes, par exemple un mécanisme de médiation flexible. Ce mécanisme devra tout particulièrement s'attacher à faciliter le règlement des différends concernant les questions relatives aux obstacles non tarifaires.

\section{Langues faisant foi}

L'accord, qui fera également foi dans toutes les langues officielles de l'UE, comportera une clause relative à la langue. 


\section{CENTRE DE RECHERCHE ET D'INFORMATION SOCIO-POLITIQUES}

Le CRISP, Centre de recherche et d'information socio-politiques, est un organisme indépendant. Ses travaux s'attachent à montrer les enjeux de la décision politique, à expliquer les mécanismes par lesquels elle s'opère, et à analyser le rôle des acteurs qui y prennent part, que ces acteurs soient politiques, économiques, sociaux, associatifs, etc.

Par ses publications, le CRISP met à la disposition d'un public désireux de comprendre la société belge des informations de haute qualité, dans un souci d'exactitude, de pertinence et de pluralisme. Son objectif est de livrer à ce public les clés d'explication du fonctionnement du système socio-politique belge et de mettre en évidence les structures réelles du pouvoir, en Belgique et dans le cadre de l'Union européenne.

Le Courrier hebdomadaire paraît au rythme de 40 numéros par an, certaines livraisons correspondant à deux numéros. Chaque livraison est une monographie consacrée à l'étude approfondie d'un aspect de la vie politique, économique ou sociale au sens large. La revue du CRISP constitue depuis 1959 une source d'information incontournable sur des sujets variés : partis politiques, organisations représentatives d'intérêts sociaux et groupes de pression divers, évolution et fonctionnement des institutions, négociations communautaires, histoire politique, groupes d'entreprises et structures du tissu économique, conflits sociaux, enseignement, immigration, vie associative et culturelle, questions environnementales, européennes, etc. C'est également dans le Courrier hebdomadaire que sont publiés les résultats des élections commentés par le CRISP.

Les auteurs publiés sont soit des chercheurs du CRISP, formés en diverses disciplines des sciences humaines, soit des spécialistes extérieurs provenant des mondes scientifique, associatif et socio-politique. Dans tous les cas, les textes sont revus avant publication par le rédacteur en chef et par un groupe d'experts sélectionnés en fonction de la problématique abordée, afin de garantir la fiabilité de l'information proposée. Cette fiabilité, ainsi que la rigoureuse objectivité du Courrier hebdomadaire, constituent les atouts principaux d'une revue dont la qualité est établie et reconnue depuis près de 60 ans.

Fondateur : Jules Gérard-Libois

Président : Vincent de Coorebyter

Équipe de recherche :

Étienne Arcq, Pierre Blaise (secrétaire général), Fabienne Collard, Vaïa Demertzis, Jean Faniel (directeur général), Christophe Goethals (coordinateur du secteur Économie), Cédric Istasse, John Pitseys, Marcus Wunderle

Conseil d'administration :

Louise-Marie Bataille, Jacques Brassinne de La Buissière (vice-président honoraire), Vincent de Coorebyter (président), Francis Delpérée, Hugues Dumont, Éric Geerkens, Nadine Gouzée, Serge Govaert, Laura Iker, Patrick Lefevre, Michel Molitor (vice-président), Solveig Pahud, Pierre Reman, Robert Tollet (vice-président), Els Witte, Paul Wynants 


\section{Derniers numéros du Courrier hebdomadaire parus}

2286-2287 Les circulaires flamandes relatives à l'emploi des langues en matière administrative Cédric Istasse

2284-2285 Le débat sur les institutions culturelles et scientifiques fédérales : une étude de l'Académie flamande

2282-2283 La réforme de l'arrondissement judiciaire de Bruxelles Bernard Blero

2280-2281 Les réformes liées à la scission de BHV : la pacification communautaire, la communauté métropolitaine et le refinancement de Bruxelles Bernard Blero

2279 La scission de la circonscription électorale de Bruxelles-Hal-Vilvorde Bernard Blero

2278 Identités, préférences et attitudes des parlementaires envers le fédéralisme belge après la sixième réforme de l'État Jérémy Dodeigne, Min Reuchamps et Dave Sinardet

2277 B-FAST et l'aide d'urgence à l'étranger Thierry Coosemans

2275-2276 La formation des gouvernements après les scrutins du 25 mai 2014 Vaïa Demertzis, Jean Faniel, Serge Govaert et Cédric Istasse

2273-2274 Le décret définissant le paysage de l'enseignement supérieur francophone ( « décret Marcourt») Jean-Émile Charlier et Michel Molitor

2271-2272 Les projets de fusion dans l'enseignement supérieur en Hainaut et dans le Luxembourg Jean-Émile Charlier et Michel Molitor

2269-2270 L'échec du projet de fusion au sein de l'Académie universitaire Louvain Michel Molitor et Jean-Émile Charlier

2268 Les dynamiques de fusion dans l'enseignement supérieur francophone de 1999 à 2009 Jean-Émile Charlier et Michel Molitor

2266-2267 La Communauté germanophone après la sixième réforme de l'État : état des lieux, débats et perspectives

Frédéric Bouhon, Christoph Niessen et Min Reuchamps

La collection intégrale du Courrier hebdomadaire est accessible sur www.cairn.info.

L'accès est gratuit pour les numéros parus avant 2011.

Découvrez notre catalogue complet incluant nos autres publications sur www.crisp.be.

Pour être informé de nos publications dès leur parution, inscrivez-vous en ligne à notre lettre d'information électronique. 